\title{
Transmutations of supersymmetry through soliton scattering, and self-consistent condensates
}

\author{
Adrián Arancibia and Mikhail S. Plyushchay \\ Departamento de Física, Universidad de Santiago de Chile, Casilla 30\%, Santiago 2, Chile \\ E-mails: adaran.phi@gmail.com,mikhail.plyushchay@usach.cl
}

\begin{abstract}
We consider the two most general families of the $(1+1)$ D Dirac systems with transparent scalar potentials, and two related families of the paired reflectionless Schrödinger operators. The ordinary $\mathcal{N}=2$ supersymmetry for such Schrödinger pairs is enlarged up to an exotic $\mathcal{N}=4$ nonlinear centrally extended supersymmetric structure, which involves two bosonic integrals composed from the Lax-Novikov operators for the stationary Korteweg-de Vries hierarchy. Each associated single Dirac system displays a proper $\mathcal{N}=2$ nonlinear supersymmetry with a non-standard grading operator. One of the two families of the first and second order systems exhibits the unbroken supersymmetry, while another is described by the broken exotic supersymmetry. The two families are shown to be mutually transmuted by applying a certain limit procedure to the soliton scattering data. We relate the topologically trivial and nontrivial transparent potentials with self-consistent inhomogeneous condensates in Bogoliubov-de Gennes and Gross-Neveu models, and indicate the exotic $\mathcal{N}=4$ nonlinear supersymmetry of the paired reflectionless Dirac systems.
\end{abstract}

\section{Introduction}

The Schrödinger and Dirac equations with reflectionless, or soliton potentials are exactly solvable. The reflectionless potentials of a general form for one-dimensional Schrödinger equation were obtained for the first time by Kay and Moses by solving the problem of a theoretical construction of a solid dielectric medium which is perfectly transparent to electromagnetic radiation [1]. Such perfectly transparent potentials appear in (1+1)-dimensional Gross-Neveu (GN) model [2]-[4], and are closely related with a nonlinear problem of selfconsistency of the Bogoliubov-de Gennes (BdG) equations [5, 6, 7]. They find applications in the description of a broad spectrum of phenomena in diverse areas of physics such as conducting polymers [8]-[13], charge fractionalization [14]-[17], and superconductivity [5]-[7], [18]-22], just to mention a few. There is also a great interest in supersymmetry associated with fermions in soliton backgrounds [23]-28].

Reflectionless potentials play a fundamental role in the theory of integrable systems. They appear as soliton solutions, particularly, to the Korteweg-de Vries (KdV) and modified 
Korteweg-de Vries (mKdV) equations. Their explicit form can be obtained by means of inverse scattering method, by Bäcklund transformation, or by Darboux-Crum transformations [1], [29]-[41]. A characteristic feature of the two last methods is a possibility to construct these potentials from simple (formal) solutions of the free particle.

In the present work we focus on the Darboux transformations. In this picture there appear the first order differential operators, which intertwine reflectionless Schrödinger, and perfectly transparent Dirac Hamiltonians. This will allow us, following the line of refs. 34, 35, 36, 42, 43, 44, 45, to study the interrelations between the exotic nonlinear supersymmetric structures emerging in the first and in the second order quantum reflectionless systems of the most general form corresponding to the $\mathrm{KdV}$ and $\mathrm{mKdV}$ solitons ${ }^{1}$. We also will observe an interesting phenomenon of transmutation of supersymmetry associated with the soliton scattering, and will relate the construction to the self-consistent inhomogeneous condensates appearing in the GN and BdG models.

A relation of the soliton potentials with the GN model [2] goes back to the famous result of Dashen, Hasslacher and Neveu [3], who found that minimizing the effective action of the model for the 'condensate function' $\sigma(x)=-g \bar{\psi} \psi$, results in the condition that the Schrödinger potentials $U_{ \pm}(x)$ given in terms of the Miura transformation [40], $U_{ \pm}(x) \equiv g^{2} \sigma^{2}(x) \pm g d \sigma(x) / d x$, have to be reflectionless. On the other hand, the Dirac system with transparent potential $\sigma(x)$ appears in the Takayama-Lin-Liu-Maki (TLM) model for conducting polymers [8], which is a continuous model for solitons in polyacetylene, where the kink and kink-antikink solutions were found [12. Though these two models have distinct physical interpretations, they are equivalent mathematically, and the methods developed in the context of the GN model were applied in the study of the TLM model [11, 9, 13]. In general, the self-consistent solutions of the GN model are related with the Ablowitz-KaupNewell-Segur hierarchy [45, 51, and by the same reason are intimately related with integrable systems in 1+1 dimensions. Particularly, some solutions to the GN model were found to be related with the breather type solutions of the sinh-Gordon and nonlinear Schrödinger equations [52].

The integrability of the equations of the $\mathrm{KdV}$ and $\mathrm{mKdV}$ hierarchies can be associated with existence of an auxiliary spectral problem given in terms of the spectral operator $H$ and the evolution generator $P_{j}$. The consistency condition appears there in the form of the equation for the Lax pair $\left(H, P_{j}\right), \frac{d H}{d t_{j}}=\left[H, P_{j}\right]$, which is equivalent to the corresponding evolution equation. For the $j$-th equation of the $\mathrm{KdV}$ hierarchy, $H=-\frac{d^{2}}{d x^{2}}+U$ is the Schrödinger operator, while $P_{j}, j=0,1, \ldots$, is an anti-Hermitian monic differential operator of the form $P_{j}=\frac{d^{2 j+1}}{d x^{2 j+1}}+a_{2 j-1} \frac{d^{2 j-1}}{d x^{2 j-1}}+\ldots+a_{0}$ with coefficient functions $a_{i}$ given in terms of the potential $U$ and its $x$-derivatives. The case of the $\mathrm{KdV}$ equation corresponds to $j=1$, and its an $n$-soliton solution $U_{n}(x, t)$ satisfies simultaneously the equation $\left[\mathcal{L}_{n}, H_{n}\right]=0$, which is the nonlinear ordinary differential equation of order $2 n+1$ in $x$ variable. This is the $n$-th stationary equation for the $\mathrm{KdV}$ hierarchy, in which $t_{1}=t$ plays a role of an external parameter. The operator $\mathcal{L}_{n}=P_{n}+\sum_{j=0}^{n-1} c_{j} P_{j}$, where $c_{j}$ are some real coefficients, is the Lax-Novikov nontrivial integral of motion for $H_{n}=-\frac{d^{2}}{d x^{2}}+U_{n}$ [32, 33, 48]. According to a

\footnotetext{
${ }^{1}$ For the earlier studies related to the appearance of the exotic extended supersymmetric structure in such class of the systems characterized by the presence of the nontrivial Lax-Novikov integral, see also Refs. [46, 47, 48, 49, 50.
} 
celebrated result of Burchnall and Chaundy [41, the square of the order $2 n+1$ differential operator $\mathcal{L}_{n}$ reduces to a certain polynomial in $H_{n}$.

One can construct the pair $\left(H_{n}, \mathcal{L}_{n}\right)$ corresponding to an $n$-soliton potential $U_{n}$ recursively, staring from the free particle case with $H_{0}=-\frac{d^{2}}{d x^{2}}$ and $\mathcal{L}_{0}=\frac{d}{d x}\left(U_{0}=0\right)$, and using the Darboux transformations. If we restrict ourselves by regular on the $x$-axis potentials, then at each step,

- i) from $U_{n}$, we construct an almost isospectral reflectionless potential $U_{n+1}$ with one more bound state in comparison with $U_{n}$, and from the Lax-Novikov integral $\mathcal{L}_{n}$ for $H_{n}=-\frac{d^{2}}{d x^{2}}+U_{n}$, we obtain the integral $\mathcal{L}_{n+1}$ for reflectionless Schrödinger system $H_{n+1}$.

The interesting point here is that having reflectionless Schrödinger potential $U_{n}$ of a general form, by applying Darboux transformation of another nature,

- ii) we can construct from $U_{n}$ another $n$-soliton potential $\tilde{U}_{n}$ to be completely isospectral to $U_{n}$, and from $\mathcal{L}_{n}$ we can obtain the corresponding integral $\tilde{\mathcal{L}}_{n}$ for $\tilde{H}_{n}$.

The latter construction can be realized by applying a certain limit procedure for soliton scattering data of the reflectionless potential $U_{n+1}$. By a similar limit procedure one can also relate $\tilde{U}_{n}$ with $U_{n-1}$, and $U_{n}$ with $U_{n-2}$. In both cases i) and ii) above, one can associate with the corresponding pairs of the reflectionless second order Hamiltonians the exotic $\mathcal{N}=4$ nonlinear supersymmetry that includes two bosonic integrals composed from Lax-Novikov integrals for the partner subsystems.

Exploiting the knowledge of the Darboux transformations for the $\mathrm{KdV}$, one can generalize the construction for the case of the $\mathrm{mKdV}$ to get the transparent Dirac systems with the multi-kink scalar potentials, and to identify for each such a single first order matrix system a proper exotic $\mathcal{N}=2$ supersymmetry. As in the Schrödinger case, the transparent Dirac multi-kink potentials are separated into the two groups: one of them is formed by topological and another by non-topological scalar potentials. The topological potentials are associated with the case i) above, and represent the configurations of $n$ kinks and $n \pm 1$ antikinks. The non-topological transparent potentials correspond to the case ii), and represent a certain superposition of $n$ kinks and $n$ antikinks. We shall show how the kinks and antikinks in transparent Dirac potentials gather together in such a way that their amplitudes and phases are fixed by supersymmetry of the paired reflectionless Schrödinger systems.

The paper is organized as follows. In Section 2 we review shortly the recursive construction of the multi-soliton Schrödinger potentials of the most general form in terms of the Darboux transformations, and describe the spectra of the corresponding reflectionless Schrödinger operators. We identify there the independent differential operators of orders 1 and $2 n$, which intertwine the neighbour in a recursive chain Schrödinger Hamiltonians $H_{n}$ and $H_{n-1}$, and find the Lax-Novikov integral of differential order $2 n+1$ for $H_{n}$. In Section 3 we describe a unique another family of the reflectionless pairs $\left(H_{n}, \tilde{H}_{n}\right)$ with completely isospectral partners, which are also intertwined by the Darboux generators to be differential operators of the same orders 1 and $2 n$, and find a certain limit procedure, related to the soliton scattering, which mutually transmutes the two indicated families of the pairs of the transparent Schrödinger systems. In Section 4 we study the exotic nonlinear supersymmetries of the two families of the Schrödinger systems composed from the reflectionless 
isospectral, $\left(H_{n}, \tilde{H}_{n}\right)$, and almost isospectral, $\left(H_{n}, H_{n-1}\right)$, pairs, and observe the transmutations between these two families through the soliton scattering. In Section 5 we study the transparent Dirac systems associated with the two families of the superextended reflectionless Schrödinger systems, where we show that each single transparent Dirac system possesses its own exotic nonlinear supersymmetry. The last Section 6 is devoted to the discussion of the obtained results and outlook. There we relate the perfectly transparent scalar Dirac potentials with the self-consistent inhomogeneous condensates appearing in the BdG and GN models, and indicate the exotic $\mathcal{N}=4$ nonlinear supersymmetry of the paired reflectionless Dirac systems.

\section{Reflectionless Schrödinger potentials and Darboux- Crum transformations}

Let $H_{n}=-d^{2} / d x^{2}+U_{n}(x)$ be a reflectionless Schrödinger system with a potential of a general $2 n$-parametric form $U_{n}(x)=U_{n}\left(x ; \kappa_{1}, \tau_{1}, \ldots, \kappa_{n}, \tau_{n}\right)$ such that $U_{n}(x) \rightarrow 0$ for $x \rightarrow \pm \infty$. Parameters $\kappa_{j}, j=1,2, \ldots, n, 0<\kappa_{1}<\ldots<\kappa_{n}$, correspond to the energy levels of the $n$ bound states, $E_{j}=-\kappa_{j}^{2}$. They also define the transmission amplitude in the scattering sector with $E=k^{2} \geq 0: t(k)=\prod_{j=1}^{n} \frac{k+i \kappa_{j}}{k-i \kappa_{j}}$, and so $|t(k)|=1$ for any real value of the wave number $k$. The parameters $\tau_{j}$ are related to the norming constants of the bound states solutions [33, 53], and their variation provides an isospectral deformation of the quantum system.

From the viewpoint of the inverse scattering method, function $U_{n}\left(x ; \kappa_{1}, \tau_{1}, \ldots, \kappa_{n}, \tau_{n}\right)$ corresponds to the instant image of the $n$-soliton solution $U_{n}(x, t)$ to the $\mathrm{KdV}$ equation $u_{t}-6 u u_{x}+u_{x x x}=0$. For large negative and positive values of time $t$ the $U_{n}(x, t)$ can be represented as a superposition of $n$ one-soliton solutions of the amplitudes $2 \kappa_{j}^{2}$ propagating to the right at the speeds $v_{j}=4 \kappa_{j}^{2}$,

$$
U_{n}(x, t)=-\sum_{j=1}^{n} 2 \kappa_{j}^{2} \operatorname{sech}^{2} \kappa_{j}\left(x-4 \kappa_{j}^{2} t-\varphi_{j}^{ \pm}\right) \quad \text { for } \quad t \rightarrow \pm \infty .
$$

The phases $\varphi_{j}^{ \pm}$defined for $t \rightarrow \pm \infty$ are given by [33, 53]

$$
\varphi_{l}^{ \pm}=\tau_{l}^{0} \pm \frac{1}{2 \kappa_{l}}\left\{\sum_{j=l+1}^{n} \log \left|\frac{\kappa_{l}+\kappa_{j}}{\kappa_{l}-\kappa_{j}}\right|-\sum_{j=1}^{l-1} \log \left|\frac{\kappa_{l}+\kappa_{j}}{\kappa_{l}-\kappa_{j}}\right|\right\},
$$

where it is implied that for $l=n$ and $l=1$ the first and, respectively, the second sum disappears. The parameter $\tau_{l}^{0}$ corresponds to the mean of the asymptotic phases, $\tau_{l}^{0}=$ $\frac{1}{2}\left(\varphi_{l}^{+}+\varphi_{l}^{-}\right)$. According to $(2.2)$, the solitons demonstrate in some sense a fermion-like behaviour: $\left|\varphi_{l}^{ \pm}\right|,\left|\varphi_{l+1}^{ \pm}\right| \rightarrow \infty$ as soon as $\kappa_{l} \rightarrow \kappa_{l+1}$. In the two-soliton case, 2.2) gives:

$$
\varphi_{1}^{ \pm}=\tau_{1}^{0} \pm \frac{1}{2 \kappa_{1}} \log \left|\frac{\kappa_{1}+\kappa_{2}}{\kappa_{1}-\kappa_{2}}\right|, \quad \varphi_{2}^{ \pm}=\tau_{2}^{0} \mp \frac{1}{2 \kappa_{2}} \log \left|\frac{\kappa_{1}+\kappa_{2}}{\kappa_{1}-\kappa_{2}}\right| .
$$

Our consideration will be based on the method of iterated Darboux transformations (or, that is the same, the Darboux-Crum transformations) [29], by which the quantum mechanical 
reflectionless system with $n$ bound states can be constructed from a free particle system $H_{0}=-\frac{d^{2}}{d x^{2}}$,

$$
H_{n}=H_{0}+U_{n}(x), \quad U_{n}=-2 \frac{d^{2}}{d x^{2}} \log \mathbb{W}_{n} .
$$

Here $\mathbb{W}_{n}$ is the Wronskian of $n$ formal (non-physical) eigenstates $\psi_{j}$ of $H_{0}, H_{0} \psi_{j}=-\kappa_{j}^{2} \psi_{j}$,

$$
\mathbb{W}_{n}=\mathbb{W}\left(\psi_{1}, \ldots, \psi_{n}\right)=\operatorname{det} \mathcal{A}, \quad \mathcal{A}_{i j}=\frac{d^{i-1}}{d x^{i-1}} \psi_{j}, \quad i, j=1, \ldots, n
$$

which are chosen as follows,

$$
\psi_{j}=\left\{\begin{array}{ll}
\cosh \left(\kappa_{j}\left(x+\tau_{j}\right)\right) & \text { for } j=\text { odd } \\
\sinh \left(\kappa_{j}\left(x+\tau_{j}\right)\right) & \text { for } j=\text { even }
\end{array}, \quad 0<\kappa_{1}<\kappa_{2}<\ldots<\kappa_{j-1}<\kappa_{n} .\right.
$$

Eigenfunctions $\Psi_{0}(x ; E) \neq \psi_{j}$ of $H_{0}, H_{0} \Psi_{0}(x ; E)=E \Psi_{0}(x ; E)$, are mapped into the eigenfunctions $\Psi_{n}(x ; E)$ of $H_{n}, H_{n} \Psi_{n}(x ; E)=E \Psi_{n}(x ; E)$, by means of the Wronskian fractions,

$$
\Psi_{n}(x ; E)=\mathbb{W}\left(\psi_{1}, \ldots, \psi_{n}, \Psi_{0}(E)\right) / \mathbb{W}_{n}
$$

The eigenfunctions in the scattering sector with $E=k^{2} \geq 0, k \geq 0$, and (not normalized) bound states with energies $E_{j}=-\kappa_{j}^{2}, j=1, \ldots, n$, of the system $H_{n}$ are given then by the relations

$$
\Psi_{n}^{ \pm}\left(k^{2}\right)=\mathbb{W}\left(\psi_{1}, \ldots, \psi_{n}, e^{ \pm i k x}\right) / \mathbb{W}_{n}, \quad \Psi_{n}\left(-\kappa_{j}^{2}\right)=\mathbb{W}\left(\psi_{1}, \ldots, \psi_{n}, \frac{d \psi_{j}}{d x}\right) / \mathbb{W}_{n}
$$

The derivative $\frac{d \psi_{j}}{d x}$ is a non-physical eigenfunction of $H_{0}$ which is linearly independent from the corresponding non-physical eigenfunction $\psi_{j}$ from $(2.6)$.

Coherently with $(2.4)$, we put $\mathbb{W}_{0}=1$ and define the prepotentials $\Omega_{n}, n=0,1, \ldots$,

$$
\Omega_{n}=-\frac{d}{d x} \log \mathbb{W}_{n} \quad \Rightarrow \quad \frac{d}{d x} \Omega_{n}=\frac{1}{2} U_{n} .
$$

Then we introduce the first order differential operators

$$
A_{n}=\frac{d}{d x}+\mathcal{W}_{n}, \quad \mathcal{W}_{n}=\Omega_{n}-\Omega_{n-1}
$$

where, particularly, $\mathcal{W}_{1}=\Omega_{1}=-\kappa_{1} \tanh \kappa_{1}\left(x+\tau_{1}\right)$. These operators and their conjugate ones factorize the reflectionless systems $H_{n-1}$ and $H_{n}$ having the $(n-1)$ - and $n$-soliton potentials $U_{n-1}\left(x ; \kappa_{1}, \tau_{1}, \ldots, \kappa_{n-1}, \tau_{n-1}\right)$ and $U_{n}\left(x ; \kappa_{1}, \tau_{1}, \ldots, \kappa_{n-1}, \tau_{n-1}, \kappa_{n}, \tau_{n}\right)$,

$$
A_{n}^{\dagger} A_{n}=H_{n-1}+\kappa_{n}^{2}, \quad A_{n} A_{n}^{\dagger}=H_{n}+\kappa_{n}^{2},
$$

and intertwine them,

$$
A_{n} H_{n-1}=H_{n} A_{n}, \quad A_{n}^{\dagger} H_{n}=H_{n-1} A_{n}^{\dagger}
$$

The operator $A_{n}$ can be presented equivalently as $A_{n}=\Psi_{n-1}^{A} \frac{d}{d x}\left(1 / \Psi_{n-1}^{A}\right)$, where $\Psi_{n-1}^{A} \equiv \frac{\mathbb{W}_{n}}{\mathbb{W}_{n-1}}$ is a nodeless non-physical eigenfunction of $H_{n-1}, H_{n-1} \Psi_{n-1}^{A}=-\kappa_{n}^{2} \Psi_{n-1}^{A}$. This function is 
a formal (exponentially blowing up at $x= \pm \infty$ ) zero mode of the first order differential operator $A_{n}, A_{n} \Psi_{n-1}^{A}=0$. Any other (physical or non-physical) eigenstate $\Psi_{n-1}(E)$ of $H_{n-1}, H_{n-1} \Psi_{n-1}(E)=E \Psi_{n-1}(E)$, is mapped by $A_{n}$ into the eigenstate of $H_{n}$,

$$
\Psi_{n}(E)=A_{n} \Psi_{n-1}(E),
$$

with the same eigenvalue, $H_{n} \Psi_{n}(E)=E \Psi_{n}(E)$.

By iteration of (2.12), reflectionless system $H_{n}$ can be related with the free particle $H_{0}$,

$$
\mathbb{A}_{n} H_{0}=H_{n} \mathbb{A}_{n}, \quad \mathbb{A}_{n}^{\dagger} H_{n}=H_{0} \mathbb{A}_{n}^{\dagger},
$$

where $\mathbb{A}_{n}$ is the differential operador of order $n$,

$$
\mathbb{A}_{n} \equiv A_{n} \ldots A_{1}
$$

In terms of (2.15), we define the differential operator of order $2 n$,

$$
\mathcal{B}_{1}=A_{1}\left(-\frac{d}{d x}+\kappa_{1}\right), \quad \mathcal{B}_{n}=\mathbb{A}_{n}\left(-\frac{d}{d x}+\kappa_{n}\right) \mathbb{A}_{n-1}^{\dagger} \quad \text { for } \quad n=2, \ldots
$$

The iteration of relations 2.12 shows that $\mathcal{B}_{n}$ and $\mathcal{B}_{n}^{\dagger}$ also intertwine reflectionless Hamiltonians $H_{n}$ and $H_{n-1}$,

$$
\mathcal{B}_{n} H_{n-1}=H_{n} \mathcal{B}_{n}, \quad \mathcal{B}_{n}^{\dagger} H_{n}=H_{n-1} \mathcal{B}_{n}^{\dagger}
$$

Unlike $A_{n}$ and $A_{n}^{\dagger}$, they do this not directly but via the 'virtual' free particle system $H_{0}$, for which the first order differential operator $\frac{d}{d x}$, appearing explicitly in the structure of $\mathcal{B}_{n}$ is an integral of motion. Instead of (2.11) we have the relations

$$
\mathcal{B}_{n} \mathcal{B}_{n}^{\dagger}=\prod_{j=1}^{n}\left(H_{n}+\kappa_{j}^{2}\right)^{2}, \quad \mathcal{B}_{n}^{\dagger} \mathcal{B}_{n}=\prod_{j=1}^{n}\left(H_{n-1}+\kappa_{j}^{2}\right)^{2} .
$$

The operator 2.15) also allows us to find a nontrivial integral for reflectionless system $H_{n}$,

$$
\mathcal{L}_{n}=\mathbb{A}_{n} p \mathbb{A}_{n}^{\dagger}, \quad \mathcal{L}_{n}^{\dagger}=\mathcal{L}_{n}, \quad\left[\mathcal{L}_{n}, H_{n}\right]=0 .
$$

This differential operator of order $2 n+1$ is the Lax-Novikov integral for the $H_{n}$. It is a Darboux-dressed form of the integral $p=-i \frac{d}{d x}$ for the free particle system $H_{0}$, which satisfies the nonlinear supersymmetry type relation

$$
\mathcal{L}_{n}^{2}=H_{n} \prod_{i=1}^{n}\left(H_{n}+\kappa_{i}^{2}\right)^{2}
$$

The property of commutativity of $\mathcal{L}_{n}$ with $H_{n}$ means that the potential $U_{n}=2 \frac{d}{d x} \Omega_{n}$ is a solution of the $n$-th member of the KdV stationary hierarchy ${ }^{2}$.

Using analogs of the integrals 2.19) for $H_{l}$ with $0<l<n$, one could try to construct the operators intertwining $H_{n-1}$ and $H_{n}$ with $n>1$ via a virtual $H_{l}$ system. In such a way we obtain, however, a combination of $\mathcal{B}_{n}$ and $A_{n}$ with a coefficient before the latter

\footnotetext{
${ }^{2}$ Note that unlike Section 1, we take $\mathcal{L}_{n}$ here in a Hermitian form.
} 
operator to be a polynomial of order $(n-1)$ in $H_{n-1}$. For instance, $-i A_{n} \mathcal{L}_{n-1}$ is the differential operator of order $2 n$, which, like $\mathcal{B}_{n}$, intertwines $H_{n-1}$ with $H_{n}$, but reduces to $-i A_{n} \mathcal{L}_{n-1}=\mathcal{B}_{n}-\kappa_{n} A_{n} \prod_{i=1}^{n-1}\left(H_{n-1}+\kappa_{i}^{2}\right)$, and so, is not a new, independent intertwining operator. At the same time note that the intertwining operators $A_{n}$ and $\mathcal{B}_{n}$, and the integral $\mathcal{L}_{n}$ are related with the Hamiltonian $H_{n}$ by the identity

$$
\mathcal{B}_{n} A_{n}^{\dagger}+i \mathcal{L}_{n}=\kappa_{n} \prod_{i=1}^{n}\left(H_{n}+\kappa_{i}^{2}\right)
$$

In conclusion of this section it is worth stressing once more that the existence of the nontrivial, order $2 n$ intertwining operator $\mathcal{B}_{n}$ in addition to the first order Darboux generator $A_{n}$ as well as of the order $2 n+1$ integral $\mathcal{L}_{n}$ originates from the fact that the reflectionless system $H_{n}$ is related to the free particle $H_{0}$ by the chain of the subsequent Darboux transformations, and the $H_{0}$ possesses a nontrivial integral of motion $p=-i \frac{d}{d x}$.

\section{Soliton scattering and Darboux transformations}

Besides the discussed pairs $\left(H_{n}, H_{n-1}\right)$ of reflectionless Schrödinger systems related by the first order Darboux intertwining operators, there is another class of such systems, for which the paired Hamiltonians are also interrelated by the first order Darboux generators. Unlike the described case, the reflectionless partners in these pairs are completely isospectral. The corresponding $n$-soliton partner potentials $U_{n}\left(x ; \kappa_{1}, \tau_{1}, \ldots, \kappa_{n}, \tau_{n}\right)$ and $U_{n}\left(x ; \kappa_{1}, \tilde{\tau}_{1}, \ldots, \kappa_{n}, \tilde{\tau}_{n}\right)$ are characterized by the same scaling parameters $\kappa_{i}, i=1, \ldots, n$, but different sets of the translation parameters correlated as follows [35]:

$$
\tau_{i}-\tilde{\tau}_{i}=\frac{1}{\kappa_{i}} \operatorname{arctanh} \frac{\kappa_{i}}{\mathcal{C}}=\frac{1}{2 \kappa_{i}} \log \frac{\mathcal{C}+\kappa_{i}}{\mathcal{C}-\kappa_{i}}
$$

where $\mathcal{C}$ is an additional real parameter such that $|\mathcal{C}|>\kappa_{n}$. A comparison of the quantities (3.1) and 2.3) indicates that (3.1) can be related somehow to the effect of the scattering of solitons. In this section we show how each indicated family of the paired reflectionless systems, with partners intertwined by the first order Darboux generators, can be transformed into another by a certain limit procedure, which admits a soliton scattering interpretation.

To this aim, we first consider the limits $\tau_{n} \rightarrow \pm \infty$ applied to the reflectionless system $H_{n}$. To study the induced deformation of the potential $U_{n}$ and superpotential $\mathcal{W}_{n}$ (the latter will play a role of the potential for an associated Dirac system), it is sufficient to investigate the limits of the prepotential $\Omega_{n}$ because of the relations $2 \frac{d}{d x} \Omega_{n}=U_{n}$, and $\Omega_{n}-\Omega_{n-1}=\mathcal{W}_{n}$. We shall demonstrate that $\Omega_{n}=-\frac{d}{d x} \log \mathbb{W}_{n} \rightarrow \tilde{\Omega}_{n-1}(\mathcal{C})-\mathcal{C}$ for $\tau_{n} \rightarrow \pm \infty$, where $\mathcal{C}= \pm \kappa_{n}$, and $\tilde{\Omega}_{n-1}$ is identical to $\Omega_{n-1}$ with $\tau_{i}, i=1, \ldots, n-1$, changed for $\tilde{\tau}_{i}=\tau_{i}-\frac{1}{2 \kappa_{i}} \log \frac{\mathcal{C}+\kappa_{i}}{\mathcal{C}-\kappa_{i}}$. From here it follows also that if we apply subsequently another limit $\kappa_{n} \rightarrow \kappa_{n-1}$, or that is the same, $\tilde{\tau}_{n-1} \rightarrow \mp \infty$, the deformed (by $\kappa$-dependent $\tau$-displacements) prepotential transforms as $\left(\tilde{\Omega}_{n-1}-\mathcal{C}\right) \rightarrow \Omega_{n-2}$. So, the effect of sending subsequently the two solitons with indices $n$ and $n-1$ to infinity in opposite directions results in disappearing of the two bound states from the spectrum, without changing the rest of the $2(n-2)$ soliton parameters in the reflectionless potential $U_{n-2}$. This corresponds to a fermion-like behaviour of solitons already mentioned below Eq. 2.2. 
In the limit $\tau_{n} \rightarrow \pm \infty$, for the prepotential $\Omega_{n}=-\left(\log \mathbb{W}\left(\psi_{1}, \cdots, \psi_{n}\right)\right)_{x}$ we find that $\Omega_{n} \rightarrow-\left(\log \mathbb{W}\left(\psi_{1}, \cdots, \psi_{n-1}, C_{n}^{ \pm} e^{ \pm \kappa_{n} x}\right)\right)_{x}$, where $C_{n}^{ \pm}=\epsilon_{n}^{ \pm} \frac{1}{2} e^{ \pm \kappa_{n} \tau_{n}}$ is an exponentially divergent multiplicative factor with $\epsilon_{n}^{+}=1$ and $\epsilon_{n}^{-}=(-1)^{n+1}$. By the Wronskian properties, we have $\mathbb{W}\left(\psi_{1}, \cdots, \psi_{n-1}, C_{n}^{ \pm} e^{ \pm \kappa_{n} x}\right)=C_{n}^{ \pm \mathbb{W}}\left(\psi_{1}, \cdots, \psi_{n-1}, e^{ \pm \kappa_{n} x}\right)$. The logarithmic derivative eliminates the $x$-independent divergent multiplicative factor $C_{n}^{ \pm}$, and in the limit $\tau_{n} \rightarrow \pm \infty$ we obtain $\Omega_{n} \rightarrow-\left(\log \mathbb{W}\left(\psi_{1}, \cdots, \psi_{n-1}, e^{ \pm \kappa_{n} x}\right)\right)_{x}$. We note now that $\mathbb{W}\left(\psi_{1}, \cdots \psi_{n-1}, e^{ \pm \kappa_{n} x}\right)=e^{ \pm \kappa_{n} x} \operatorname{det}\left\|W_{n}^{\natural}\right\|$, where

$$
\left\|W_{n}^{\natural}\right\|=\left(\begin{array}{ccccc}
\operatorname{ch} \kappa_{1} x_{1} & \operatorname{sh} \kappa_{2} x_{2} & \ldots & \psi_{n-1} & 1 \\
\kappa_{1} \operatorname{sh} \kappa_{1} x_{1} & \kappa_{2} \operatorname{ch} \kappa_{2} x_{2} & & \partial_{x} \psi_{n-1} & \pm \kappa_{n} \\
\vdots & & \ddots & \vdots & \vdots \\
\partial_{x}^{n-1} \operatorname{ch} \kappa_{1} x_{1} & \partial_{x}^{n-1} \operatorname{sh} \kappa_{2} x_{2} & \ldots & \partial_{x}^{n-1} \psi_{n-1} & ( \pm 1)^{n-1} \kappa_{n}^{n-1}
\end{array}\right),
$$

and $x_{i} \equiv x+\tau_{i}$. By changing the rows $L_{j}, j=1, \ldots, n-1$, of the matrix (3.2) for the linear combinations: $L_{j} \rightarrow \kappa_{n} L_{j} \mp L_{j+1}$, we find that $\left(\log \operatorname{det}\left\|W_{n}^{\natural}\right\|\right)_{x}=\left(\log \operatorname{det}\left\|W_{n}\right\|\right)_{x}$ where

$$
\left\|\hat{W}_{n}\right\|=\left(\begin{array}{ccccc}
\mathrm{Ch}_{1}^{\mp} & \mathrm{Sh}_{2}^{\mp} & \mathrm{Ch}_{3}^{\mp} & \ldots & 0 \\
\kappa_{1} \mathrm{Sh}_{1}^{\mp} & \kappa_{2} \mathrm{Ch}_{2}^{\mp} & \kappa_{3} \mathrm{Sh}_{3}^{\mp} & \ldots & 0 \\
\kappa_{1}^{2} \mathrm{Ch}_{1}^{\mp} & \kappa_{2}^{2} \mathrm{Sh}_{2}^{\mp} & \kappa_{3}^{2} \mathrm{Ch}_{3}^{\mp} & \cdots & 0 \\
\cdot & \cdot & \ldots & \cdot & \\
\partial_{x}^{n-1} \cosh \kappa_{1} x_{1} & \partial_{x}^{n-1} \sinh \kappa_{2} x_{2} & \partial_{x}^{n-1} \cosh \kappa_{3} x_{3} & \cdots & ( \pm 1)^{n-1} \kappa_{n}^{n-1}
\end{array}\right) .
$$

Here we denote $\mathrm{Ch}_{i}^{\mp}=\kappa_{n} \cosh \kappa_{i} x_{i} \mp \kappa_{i} \sinh \kappa_{i} x_{i}$ and $\mathrm{Sh}_{i}^{\mp}=\kappa_{n} \sinh \kappa_{i} x_{i} \mp \kappa_{i} \cosh \kappa_{i} x_{i}$, $i=1, \ldots, n-1$, where the signs - and + correspond to the limits $\tau_{n} \rightarrow+\infty$ and $\tau_{n} \rightarrow-\infty$, respectively. These functions can be represented equivalently as $\mathrm{Ch}_{i}^{\mp}=\sqrt{\kappa_{n}^{2}-\kappa_{i}^{2}} \cosh \kappa_{i}(x+$ $\left.\tau_{i} \mp \varphi_{i}\right)$ and $\mathrm{Sh}_{i}^{\mp}=\sqrt{\kappa_{n}^{2}-\kappa_{i}^{2}} \sinh \kappa_{i}\left(x+\tau_{i} \mp \varphi_{i}\right)$, where $\varphi_{i}=\frac{1}{2 \kappa_{i}} \log \frac{\kappa_{n}+\kappa_{i}}{\kappa_{n}-\kappa_{i}}, i=1, \ldots, n-1$. As a consequence, we find that $\left(\log \mathbb{W}\left(\psi_{1}, \cdots, \psi_{n-1}, e^{ \pm \kappa_{n} x}\right)\right)_{x}= \pm \kappa_{n}+\left(\log \mathbb{W}\left(\tilde{\psi}_{1}, \cdots, \tilde{\psi}_{n-1}\right)\right)_{x}$, where $\tilde{\psi}_{i}$ is identical to $\psi_{i}$ but with $\tau_{i}, i=1, \ldots, n-1$, changed for

$$
\tilde{\tau}_{i}=\tau_{i} \mp \frac{1}{2 \kappa_{i}} \log \frac{\kappa_{n}+\kappa_{i}}{\kappa_{n}-\kappa_{i}} \quad \text { for } \quad \tau_{n} \rightarrow \pm \infty,
$$

that translates finally into the transformation $\Omega_{n} \underset{\tau_{n} \rightarrow \pm \infty}{\longrightarrow} \tilde{\Omega}_{n-1} \mp \kappa_{n}$. Note that $\tilde{\tau}_{i}-\tau_{i}$ given by (3.4) corresponds to the change of the phase in the two-soliton scattering given by the first relation in (2.3), with indexes 1 and 2 changed for $i$ and $n$, respectively.

In the limit $\tau_{n} \rightarrow+\infty$ we find that

$$
A_{n}=\frac{d}{d x}+\mathcal{W}_{n} \rightarrow \frac{d}{d x}-\Delta_{n-1}\left(\kappa_{n}\right)=-X_{n-1}^{\dagger}\left(\kappa_{n}\right),
$$

where

$$
X_{n-1}=\frac{d}{d x}+\Delta_{n-1}, \quad \Delta_{n-1}\left(\kappa_{n}\right)=\Omega_{n-1}-\tilde{\Omega}_{n-1}\left(\kappa_{n}\right)+\kappa_{n} .
$$

The subsequent application of the limit $\kappa_{n} \rightarrow \kappa_{n-1}$ gives

$$
X_{n-1}\left(\kappa_{n}\right) \rightarrow A_{n-1}, \quad \tilde{A}_{n-1} \rightarrow X_{n-2}\left(\kappa_{n-1}\right),
$$


where the first order operator $\tilde{A}_{n-1}$ is of the same form as $A_{n-1}$ but with the parameters $\tau_{i}$ changed for $\tilde{\tau}_{i}=\tau_{i}-\frac{1}{2 \kappa_{i}} \log \frac{\mathcal{C}+\kappa_{i}}{\mathcal{C}-\kappa_{i}}$. The relations corresponding to the limit $\tau_{n} \rightarrow-\infty$ can be written down explicitly in a similar way.

Since the $n$-soliton potentials are given by the relation $U_{n}=2 \frac{d}{d x} \Omega_{n}$, by taking the limit $\tau_{n} \rightarrow+\infty$ we eliminate the bound state with $E_{n}=-\kappa_{n}^{2}$ from the spectrum of $H_{n}$, and obtain a new Hamiltonian with $(n-1)$ bound states, which we call $\tilde{H}_{n-1}$. This hamiltonian is isospectral to $H_{n-1}$ but each soliton in it is displaced with a phase dependent on $\kappa_{n}$,

$$
H_{n}\left(\tau_{i}\right) \underset{\tau_{n} \rightarrow+\infty}{\longrightarrow} H_{n-1}\left(\tilde{\tau}_{i}\right) \equiv \tilde{H}_{n-1}\left(\kappa_{n}\right), \quad \tilde{\tau}_{i}=\tau_{i}-\frac{1}{2 \kappa_{i}} \log \frac{\kappa_{n}+\kappa_{i}}{\kappa_{n}-\kappa_{i}}
$$

The limit $\tau_{n} \rightarrow-\infty$ corresponds to the change of $\kappa_{n}$ for $-\kappa_{n}$ in (3.8). In general, from the viewpoint of $\tilde{H}_{n-1}$, the $\kappa_{n}$ (or $-\kappa_{n}$ ) is just an additional parameter, and from now on we call $\tilde{H}_{n-1} \equiv \tilde{H}_{n-1}(\mathcal{C})$, assuming for the sake of definiteness that $\mathcal{C}>\kappa_{n-1}$.

On the other hand both the Hamiltonians $H_{n}$ in the limit $\kappa_{n} \rightarrow \kappa_{n-1}$ and $\tilde{H}_{n-1}$ in the limit $\mathcal{C} \rightarrow \kappa_{n-1}$ correspond to a Hamiltonian $H_{n-2}$,

$$
H_{n} \underset{\kappa_{n} \rightarrow \kappa_{n-1}}{\longrightarrow} H_{n-2}, \quad \tilde{H}_{n-1}(\mathcal{C}) \underset{\mathcal{C} \rightarrow \kappa_{n-1}}{\longrightarrow} H_{n-2} .
$$

As analogs of factorizations (2.11) we obtain

$$
X_{n}^{\dagger} X_{n}=\tilde{H}_{n}+\mathcal{C}^{2}, \quad X_{n} X_{n}^{\dagger}=H_{n}+\mathcal{C}^{2},
$$

where $X_{n}$ is defined in (3.6) with index $n-1$ changed for $n$, and it is assumed here that $\mathcal{C}^{2}>\kappa_{n}^{2}$. In correspondence with $3.10, X_{n}$ and $X_{n}^{\dagger}$ not only factorize the isospectral Hamiltonians, but also intertwine them, $X_{n} \tilde{H}_{n}=H_{n} X_{n}, X_{n}^{\dagger} H_{n}=\tilde{H}_{n} X_{n}^{\dagger}$. We also have the factorization relations

$$
\tilde{A}_{n} \tilde{A}_{n}^{\dagger}=\tilde{H}_{n}+\kappa_{n}^{2}, \quad \tilde{A}_{n}^{\dagger} \tilde{A}_{n}=\tilde{H}_{n-1}+\kappa_{n}^{2}
$$

Using these last relations, one can construct the generators which intertwine $\tilde{H}_{n}$ and $H_{n}$, being the differential operators of the order $2 n$,

$$
\mathcal{Y}_{n}=\mathbb{A}_{n} \tilde{\mathbb{A}}_{n}^{\dagger}, \quad \mathcal{Y}_{n}^{\dagger}=\tilde{\mathbb{A}}_{n} \mathbb{A}_{n}^{\dagger},
$$

$\mathcal{Y}_{n} \tilde{H}_{n}=H_{n} \mathcal{Y}_{n}, \mathcal{Y}_{n}^{\dagger} H_{n}=\tilde{H}_{n} \mathcal{Y}_{n}^{\dagger}$, where $\tilde{\mathbb{A}}_{n}$ is defined as in 2.15 but with $A_{i}$ changed for $\tilde{A}_{i}$.

Another pair of important identities is

$$
A_{n} X_{n-1}=X_{n} \tilde{A}_{n}, \quad X_{n}^{\dagger} A_{n}=\tilde{A}_{n} X_{n-1}^{\dagger} .
$$

The operators appearing in the first equality intertwine the Hamiltonians $\tilde{H}_{n-1}$ and $H_{n}$, $\left(A_{n} X_{n-1}\right) \tilde{H}_{n-1}=H_{n}\left(A_{n} X_{n-1}\right),\left(X_{n} \tilde{A}_{n}\right) \tilde{H}_{n-1}=H_{n}\left(X_{n} \tilde{A}_{n}\right)$, and the equal operators from the other relation intertwine in a similar manner $H_{n-1}$ and $\tilde{H}_{n}$. The Hermitian conjugate form of the operators from (3.13) intertwine the indicated pairs of the Hamiltonians in the opposite direction. The relations in 3.13 are equivalent to the identity

$$
\left(\mathcal{C}+\Omega_{n-1}-\tilde{\Omega}_{n}\right)\left(\Omega_{n}-\tilde{\Omega}_{n}-\Omega_{n-1}+\tilde{\Omega}_{n-1}\right)=\left(\tilde{\Omega}_{n}-\Omega_{n-1}\right)_{x}
$$


which, in turn, is reduced to trigonometric identities [35]. In the limit $\tau_{n} \rightarrow \infty$, we find then that the intertwining between $H_{n-1}$ and $H_{n}$ operator $\mathcal{B}_{n}$, see Eq. (2.17), transforms into

$$
\mathcal{B}_{n} \rightarrow\left(\tilde{H}_{n-1}\left(\kappa_{n}\right)+\kappa_{n}^{2}\right) \mathcal{Y}_{n-1}^{\dagger}\left(\kappa_{n}\right)-2 \kappa_{n}\left(\prod_{i=1}^{n-1}\left(\tilde{H}_{n-1}\left(\kappa_{n}\right)+\kappa_{i}^{2}\right)\right) X_{n-1}^{\dagger}\left(\kappa_{n}\right)
$$

This is a reducible intertwining operator for a pair $H_{n-1}$ and $\tilde{H}_{n-1}$. From 3.15) we extract the irreducible operators $\mathcal{Y}_{n-1}^{\dagger}$ and $X_{n-1}^{\dagger}\left(\kappa_{n}\right)$ which intertwine the Hamiltonians $H_{n-1}$ and $\tilde{H}_{n-1}$. At the same time, for the Lax-Novikov integral $\mathcal{L}_{n}$ we have

$$
\mathcal{L}_{n} \rightarrow\left(\tilde{H}_{n-1}\left(\kappa_{n}\right)+\kappa_{n}^{2}\right) \tilde{\mathcal{L}}_{n-1}
$$

that provides us with the irreducible nontrivial integral $\tilde{\mathcal{L}}_{n-1}$ for $\tilde{H}_{n-1}$,

Figures 1 and 2 illustrate different limits for superpotentials $\mathcal{W}_{n}$ and $\Delta_{n}$, while figures 3 and 4 show the transformations between potentials $U_{n}$ and $\tilde{U}_{n}$.

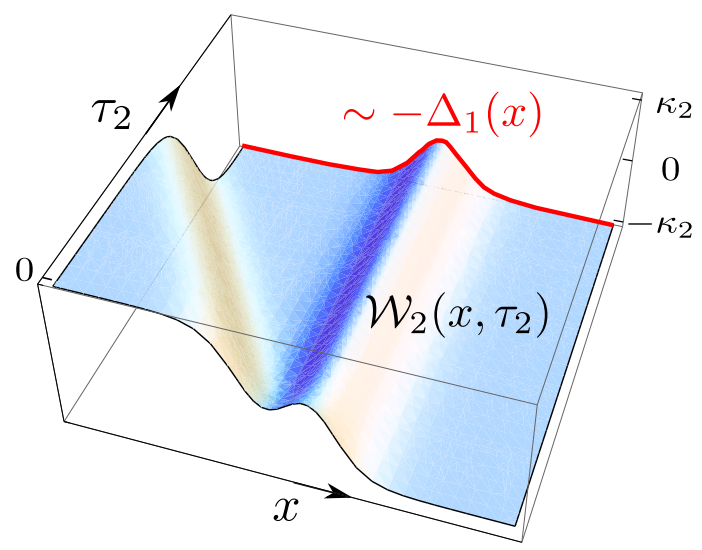

Figure 1: In the limit $\tau_{n} \rightarrow \infty$, a topologically nontrivial superpotential $\mathcal{W}_{n}$ (being also the corresponding scalar Dirac potential) with asymptotic behaviour $\lim _{x \rightarrow-\infty} \mathcal{W}_{n}(x)=$ $-\lim _{x \rightarrow+\infty} \mathcal{W}_{n}(x)=\kappa_{n}>0$ transforms (asymptotically) into a topologically trivial superpotential $-\Delta_{n-1}$ such that $\lim _{x \rightarrow-\infty} \Delta_{n-1}(x)=\lim _{x \rightarrow+\infty} \Delta_{n-1}(x)=\kappa_{n}>0$. This corresponds to sending the $n$-th kink to $x=-\infty$. The figure corresponds to the case $n=2$, and shows the superpotential $\mathcal{W}_{2}$ as a function of $x$ and $\tau_{2}$.

We have considered the limit when the translation parameter $\tau_{n}$ in the $n$-soliton potential $U_{n}$ tends to infinity. It is interesting to see what happens with reflectionless system $H_{n}$ when we take the limit $\tau_{j} \rightarrow \pm \infty$ with $j<n$. Considering the same procedure as in the case $j=n$, we find that the prepotential $\Omega_{n}$ changes for $\Omega^{\prime}{ }_{n-1}$, in which instead of (3.4) the arguments $\tau_{i}$ are replaced by

$$
\tau_{i}^{\prime}=\left\{\begin{array}{cl}
\tau_{i} \mp \frac{1}{2 \kappa_{i}} \log \frac{\kappa_{j}+\kappa_{i}}{\kappa_{j}-\kappa_{i}} & \text { for } i<j \\
\tau_{i} \pm \frac{1}{2 \kappa_{i}}\left(\log \frac{\kappa_{j}+\kappa_{i}}{\kappa_{i}-\kappa_{j}}+i \pi\right) & \text { for } i>j
\end{array}\right.
$$

\footnotetext{
${ }^{3}$ The questions of redundancy of nonlinear supersymmetric algebra in a general context were studied in [50], see also the recent review [54]. The very nontrivial picture of redundancy and transmutations appearing in the completely isospectral supersymmetric pairs of reflectionless systems was investigated in detail in 34, 35.
} 


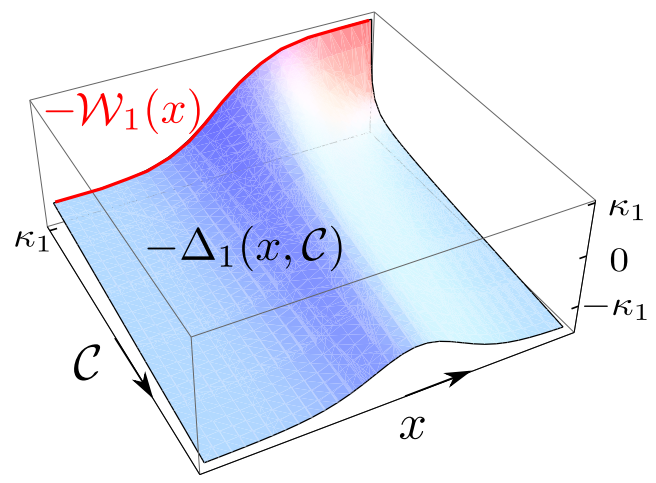

Figure 2: A topologically trivial superpotential $\Delta_{n}$ transforms into a topologically nontrivial superpotential $\mathcal{W}_{n}$ through the limit $\left|\tilde{\tau}_{n}\right| \rightarrow \infty$, which is equivalent to the limit $\mathcal{C}^{2} \rightarrow \kappa_{n}^{2}$. The figure illustrates the case when the kink-antikink Dirac potential with $n=1$ transforms in the limit $\mathcal{C} \rightarrow \kappa_{1}$ into the antikink potential.

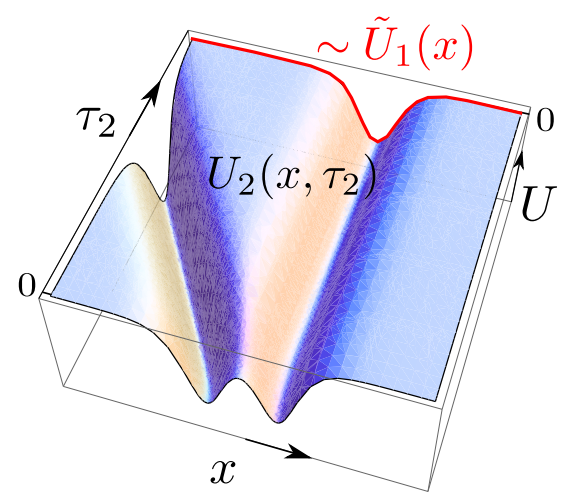

Figure 3: For the particular case of $n=2$, the figure illustrates the transformation of the Schrödinger $n$-soliton potential $U_{n}$ into the $(n-1)$-soliton potential $\tilde{U}_{n-1}$ in the limit $\tau_{n} \rightarrow \infty$.

For $i>j$ we have $\cosh \kappa_{i}\left(x+\tau_{i}^{\prime}\right)= \pm i \sinh \kappa_{i}\left(x+\hat{\tau}_{i}\right), \sinh \kappa_{i}\left(x+\tau_{i}^{\prime}\right)= \pm i \cosh \kappa_{i}\left(x+\hat{\tau}_{i}\right)$, where

$$
\hat{\tau}_{i}=\tau_{i} \mp \frac{1}{2 \kappa_{i}} \log \left|\frac{\kappa_{j}+\kappa_{i}}{\kappa_{j}-\kappa_{i}}\right| .
$$

The effect of the limit $\tau_{j} \rightarrow \pm \infty$ results then in the reduction of the reflectionless system $H_{n}\left(x ; \kappa_{1}, \tau_{1}, \ldots, \kappa_{n}, \tau_{n}\right)$ into the reflectionless system $\hat{H}_{n-1}$, where the latter Hamiltonian is given by the set of parameters $\kappa_{i}$ and $\hat{\tau}_{i}$ with $i=1, \ldots, j-1, j+1, \ldots, n$. It is also easy to check that the application of the limit $\kappa_{j} \rightarrow \kappa_{j+1}$ with $j$ taking one of the values from the set $1, \ldots, n-1$, transforms $H_{n}$ into $H_{n-2}$, where the latter reflectionless Hamiltonian is characterized by the parameters $\kappa_{i}$ and $\tau_{i}$ with $i=1, \ldots, j-1, j+1, \ldots, n$. The same effect can be obtained if we apply subsequently two limits, first $\tau_{j} \rightarrow+\infty$ (or $\tau_{j} \rightarrow-\infty$ ) and then $\hat{\tau}_{j-1} \rightarrow-\infty$ (or $\hat{\tau}_{j-1} \rightarrow+\infty$ ), i.e. sent the soliton $j$ and the transformed one with index $j-1$ to infinity in the opposite directions.

Note here that applying appropriately the described limits with $\tau_{j}$ tending to $+\infty$ or $-\infty$, 


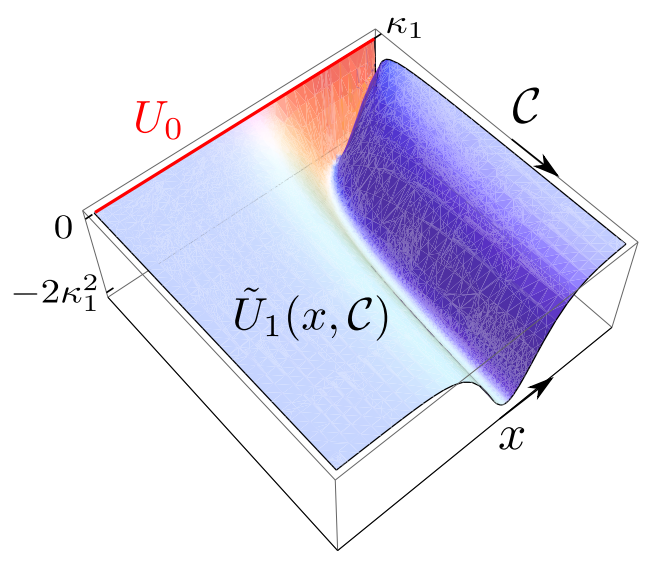

Figure 4: As an illustration for the second limit in (3.9), it is shown the transformation of the one-soliton potential $\tilde{U}_{1}(x, \mathcal{C})$ into the zero potential of the free particle case in the limit $\mathcal{C} \rightarrow \kappa_{1}$. Note that in another limit $\mathcal{C} \rightarrow \infty$, we have $\tilde{H}_{n} \rightarrow H_{n}$, but the intertwining operator $X_{n}$ blows up. Changing $X_{n}$ for the rescaled operator $\hat{X}_{n}=\frac{1}{\mathcal{C}} X_{n}$, we get in the indicated limit the trivial operator, $\hat{X}_{n} \rightarrow 1$, as an intertwiner between the two identical copies of the reflectionless Schrödinger Hamiltonian $H_{n}$.

we can reproduce exactly the phases from (2.2), which correspond to the soliton scattering picture in the $n$-soliton solution for the KdV equation. Indeed, let us fix index $i=l$, where $1 \leq l \leq n$. For the sake of generality, assume that $1<l<n$. Now, let us take a limit $\tau_{n} \rightarrow+\infty$. The displaced value of $\tau_{l}$ will be given by the upper sign case of Eq. (3.18) with $i=l$ and $j=n$. Then we send subsequently to $+\infty$ the soliton indexed by $j=n-1$, then $j=n-2$, etc., till $j=l+1$. Repeating analogous procedure with sending to $-\infty$ first the soliton with $j=1$, then with $j=2$, etc., till $j=l-1$, the resulting changed translation parameter will be given exactly by Eq. 2.2 corresponding to the case $t \rightarrow-\infty$ with $\tau_{l}^{0}$ changed for our initial value $\tau_{l}$. The sign minus in the limit $t \rightarrow-\infty$ (in comparison with the sign in the limit $\tau_{n} \rightarrow+\infty$ ) is associated with the minus sign appearing in Eq. (2.1) before the term $4 \kappa_{j}^{2} t$.

Considering the pairs of reflectionless Hamiltonians, $\left(H_{n}, H_{n-1}\right)$ or $\left(H_{n}, \tilde{H}_{n}\right)$, the partners of which are related by the first order Darboux intertwining generators, we shall see below that the limits $\tau_{n} \rightarrow \pm \infty$ induce the transmutation of the type of the supersymmetry, interchanging the cases of the unbroken and broken supersymmetries. On the other hand, the application of the limits $\tau_{j} \rightarrow \pm \infty$ with $j<n$ reduces only the number of the bound states in the partner Hamiltonians, but does not change the type of the corresponding supersymmetry of the extended reflectionless system.

The difference of the corresponding supersymmetries in the two cases can be explained by different nature of the first order Darboux intertwining generators. In the case of the pairs $\left(H_{n}, H_{n-1}\right)$, the intertwining generators $A_{n}$ and $A_{n}^{\dagger}$ are constructed in terms of the superpotential $\mathcal{W}_{n}$, see Eq. 2.10$]$, for which $\mathcal{W}_{n}(x) \rightarrow \mp \kappa_{n}$ for $x \rightarrow \pm \infty$. This superpotential takes asymptotically the constant values of the opposite signs, and is topologically nontrivial. The Witten index for such extended system takes nonzero value, and the associated first order supersymmery (see the next section), is unbroken [24, 25]. The isospectral 
partners in the pairs $\left(H_{n}, \tilde{H}_{n}\right)$ are intertwined by the first order Darboux generators $X_{n}$ and $X_{n}^{\dagger}$, constructed in terms of the superpotential $\Delta_{n}(\mathcal{C})$, see Eq. 3.6 with $n-1$ changed for $n$. Since $\lim _{x \rightarrow+\infty} \Delta_{n}=\lim _{x \rightarrow-\infty} \Delta_{n}=\mathcal{C}$ with $\mathcal{C}^{2}>\kappa_{n}^{2}>0$, the superpotential $\Delta_{n}(\mathcal{C})$ is topologically trivial, and the corresponding first order supersymmetry will be broken in correspondence with zero value of the Witten index.

\section{Exotic supersymmetry of reflectionless systems with the first order supercharges}

Consider now an extended $2 \times 2$ matrix Hamiltonian $\mathcal{H}=\operatorname{diag}\left(H, H^{\prime}\right)$ with $H$ and $H^{\prime}$ to be reflectionless systems, and identify $\Gamma=\sigma_{3}$ as a $\mathbb{Z}_{2}$-grading operator. As it was shown in [34, in general case such a system is characterized by exotic nonlinear supersymmetry with two pairs of supercharges, which are the matrix higher order derivative operators of the anti-diagonal form, constructed from the Darboux-Crum intertwiners. The symmetry structure of $\mathcal{H}$ also has to include two higher order Lax-Novikov integrals of the subsystems $H$ and $H^{\prime}$. Within this class of the extended reflectionless systems, there exist two special families, for which a pair of fermionic integrals are the first order matrix differential operators of the form $S_{a}=S_{a}^{\dagger}=\sigma_{a} \operatorname{diag}\left(D, D^{\dagger}\right), a=1,2$, which satisfy the relations $\left[S_{a}, \mathcal{H}\right]=0$ and $\left\{S_{a}, S_{b}\right\}=2 \delta_{a b}(\mathcal{H}+$ const $)$. The operators $D$ and $D^{\dagger}$ in this case not only intertwine the Hamiltonian operators $H$ and $H^{\prime}$, but also factorize them, $H=D^{\dagger} D+$ const and $H^{\prime}=D D^{\dagger}+$ const 4 .

Without loss of generality one can choose $H=H_{n}$ to be reflectionless Hamiltonian with $n$-soliton potential. Then there are only three possibilities to choose $H^{\prime}$ such that $H$ and $H^{\prime}$ can be related by the intertwining operators of the first order. These possibilities are $H^{\prime}=H_{n-1}, H^{\prime}=H_{n+1}$, or $H^{\prime}=\tilde{H}_{n}(\mathcal{C})$. The trivial case of a free particle, $H_{0}$, is exceptional: for it there are only two possibilities, $H^{\prime}=H_{1}$ and $H^{\prime}=H_{0}$, due to the translation invariance of $H_{0}$.

Having this picture in mind, we first consider a class of the extended reflectionless $(2 n+1)$ parametric systems composed from isospectral Hamiltonians each having $n$ bound states. It is convenient to shift the Hamiltonian operators for an additive constant term, and take

$$
\breve{\mathcal{H}}_{n}=\left(\begin{array}{cc}
H_{n}^{\mathcal{C}} & 0 \\
0 & \tilde{H}_{n}^{\mathcal{C}}
\end{array}\right)
$$

as the extended Hamiltonian, where $H_{n}^{\mathcal{C}}=H_{n}+\mathcal{C}^{2}, \tilde{H}_{n}^{\mathcal{C}}=\tilde{H}_{n}+\mathcal{C}^{2}$. A real constant $\mathcal{C}$ is restricted here by the condition $\mathcal{C}^{2}>\kappa_{n}^{2}$, and $\tilde{H}_{n}=\tilde{H}_{n}(\mathcal{C})$ is the reflectionless system isospectral to $H_{n}$ but with the parameters $\tau_{i}$ in the $n$-soliton potential changed for the shifted set of translation parameters $\tilde{\tau}_{i}$ given by equation (3.1). The spectra of the isospectral partner Hamiltonians are

$$
\sigma\left(H_{n}^{\mathcal{C}}\right)=\sigma\left(\tilde{H}_{n}^{\mathcal{C}}\right)=\mathcal{C}^{2}-\kappa_{n}^{2} \cup \ldots \cup \mathcal{C}^{2}-\kappa_{1}^{2} \cup\left[\mathcal{C}^{2}, \infty\right)
$$

Each discrete energy level $\mathcal{C}^{2}-\kappa_{i}^{2}, i=1, \ldots, n$, of the extended system (4.1), as well as the energy level $E=\mathcal{C}^{2}$ at the edge of the continuous part of the spectrum are doubly

\footnotetext{
${ }^{4}$ The supercharges, which are the higher order derivative operators, factorize certain polynomials of the partner Hamiltonians in correspondence with relations of the form 2.18.
} 
degenerate. At the same time, each energy level inside the conduction band $\left(\mathcal{C}^{2}, \infty\right)$ of $\breve{\mathcal{H}}_{n}$ is four-fold degenerate. The set of the nontrivial integrals of motion (in addition to the trivial integral $\Gamma=\sigma_{3}$ ) of the supersymmetric system (4.1) consists of the two matrix differential operators of the first order composed from the Darboux intertwining generators of the form (3.6) (with index $n-1$ changed for $n$ ),

$$
\breve{\mathcal{S}}_{n, 1}=\left(\begin{array}{cc}
0 & X_{n} \\
X_{n}^{\dagger} & 0
\end{array}\right), \quad \breve{\mathcal{S}}_{n, 2}=i \sigma_{3} \breve{\mathcal{S}}_{n, 1} .
$$

We have also two matrix integrals to be differential operators of the order $2 n$ constructed from the intertwines 3.12 ,

$$
\breve{\mathcal{Q}}_{n, 1}=\left(\begin{array}{cc}
0 & \mathcal{Y}_{n} \\
\mathcal{Y}_{n}^{\dagger} & 0
\end{array}\right), \quad \breve{\mathcal{Q}}_{n, 2}=i \sigma_{3} \breve{\mathcal{Q}}_{n, 1}
$$

In addition, the system is characterized by the two diagonal matrix integrals constructed from the Lax-Novikov integrals $(2.19)$ of the subsystems, which are the differential operators of the order $2 n+1$,

$$
\breve{\mathcal{P}}_{n, 1}=\left(\begin{array}{cc}
\mathcal{L}_{n} & 0 \\
0 & \tilde{\mathcal{L}}_{n}
\end{array}\right), \quad \breve{\mathcal{P}}_{n, 2}=\sigma_{3} \breve{\mathcal{P}}_{n, 1} .
$$

With the chosen $\mathbb{Z}_{2}$-grading operator $\Gamma=\sigma_{3}$, operators $(4.2)$ and $(4.3)$ are identified as the fermionic integrals, and (4.4) are identified as the bosonic generators. They, together with the Hamiltonian $\breve{\mathcal{H}}_{n}$, generate the exotic superalgebra, whose nonzero (anti)-commutation relations are given by

$$
\begin{gathered}
\left\{\breve{\mathcal{S}}_{a}, \breve{\mathcal{S}}_{b}\right\}=2 \delta_{a b} \breve{\mathcal{H}}, \quad\left\{\breve{\mathcal{Q}}_{a}, \breve{\mathcal{Q}}_{b}\right\}=2 \delta_{a b} \breve{\mathbb{P}}^{2}, \quad\left\{\breve{\mathcal{S}}_{a}, \breve{\mathcal{Q}}_{b}\right\}=2 \delta_{a b} \mathcal{C} \breve{\mathbb{P}}+2 \epsilon_{a b} \breve{\mathcal{P}}_{1}, \\
{\left[\breve{\mathcal{P}}_{2}, \breve{\mathcal{S}}_{a}\right]=2 i\left(\breve{\mathcal{H}} \breve{\mathcal{Q}}_{a}-\mathcal{C} \breve{\mathbb{P}}_{a}\right), \quad\left[\breve{\mathcal{P}}_{2}, \breve{\mathcal{Q}}_{a}\right]=2 i \breve{\mathbb{P}}\left(\mathcal{C} \breve{\mathcal{Q}}_{a}-\breve{\mathbb{P}} \breve{\mathcal{S}}_{a}\right)}
\end{gathered}
$$

where $\breve{\mathbb{P}}_{n}=\prod_{j=1}^{n}\left(\breve{\mathcal{H}}_{n}-\mathcal{C}^{2}+\kappa_{j}^{2}\right)$, and to simplify the expressions we omitted the index $n$ in 4.5), 4.6). Though our construction with the two Schrödinger subsystems $H_{n}^{\mathcal{C}}$ and $\tilde{H}_{n}^{\mathcal{C}}$ corresponds to the usual $\mathcal{N}=2$ supersymmetry generated by the two supercharges $\mathcal{S}_{n, a}$ to be matrix differential operators of the first order, we have obtained the exotic supersymmetric structure with the two additional supercharges $\breve{\mathcal{Q}}_{n, a}$ to be the higher order differential operators. In addition, being the extended reflectionless system, it also possesses two bosonic integrals of motion. The peculiarity of the present exotic supersymmetric structure is that the bosonic integral $\breve{\mathcal{P}}_{n, 1}$ commutes with all the other integrals of motion, and plays a role of the central charge operator of the nonlinear superalgebra $\left.\right|^{5}$. Another bosonic integral $\breve{\mathcal{P}}_{n, 2}$ realizes a rotation of the pairs of the supercharges $\breve{\mathcal{S}}_{n, a}$ and $\breve{\mathcal{Q}}_{n, a}$ by means of the commutation relations 4.6) with the Hamiltonian-dependent structure coefficients.

Since the doublet of the ground states of $\breve{\mathcal{H}}_{n}$ has positive energy $\mathcal{C}^{2}-\kappa_{n}^{2}>0$, the first order supercharges $\breve{\mathcal{S}}_{n, a}$ do not annihilate them either, and the $\mathcal{N}=2$ Lie sub-superalgebra generated by $\breve{\mathcal{S}}_{n, a}$ and $\breve{\mathcal{H}}_{n}$ corresponds to the phase of the broken supersymmetry. At the same time, according to Eq. 2.20), the doublet of the ground states is annihilated by the

\footnotetext{
${ }^{5}$ This is not so in a general case of the extended system composed from the two $n$-soliton Schrödinger subsystems, see ref. [34].
} 
bosonic integrals $\breve{\mathcal{P}}_{n, a}$. Due to the second relation from 4.5 , they are also annihilated by the higher order supercharges $\breve{\mathcal{Q}}_{n, a}$. One can conclude therefore that the obtained exotic nonlinear $\mathcal{N}=4$ supersymmetry of the extended reflectionless system $\breve{\mathcal{H}}_{n}$ is partially broken.

Let us apply now the limit $\mathcal{C}^{2} \rightarrow \kappa_{n}^{2}$, associated with the soliton scattering, to the system $\breve{\mathcal{H}}_{n}$. For the sake of definiteness, let us assume that $\mathcal{C}$ is positive, and consider the limit $\mathcal{C} \rightarrow \kappa_{n}$, which corresponds to the limit $\tilde{\tau}_{n} \rightarrow-\infty$ for the subsystem $\tilde{H}_{n}^{\mathcal{C}}$. In this limit, the Hamiltonian (4.1) and integrals of motion are transformed into

$$
\begin{gathered}
\mathcal{H}_{n}=\left(\begin{array}{cc}
H_{n}^{\kappa_{n}} & 0 \\
0 & H_{n-1}^{\kappa_{n}}
\end{array}\right), \quad \mathcal{S}_{n, 1}=\left(\begin{array}{cc}
0 & A_{n} \\
A_{n}^{\dagger} & 0
\end{array}\right), \\
\mathcal{Q}_{n, 1}=\left(\begin{array}{cc}
0 & \mathcal{B}_{n} \\
\mathcal{B}_{n}^{\dagger} & 0
\end{array}\right), \quad \mathcal{P}_{n, 1}=\left(\begin{array}{cc}
\mathcal{L}_{n} & 0 \\
0 & H_{n-1}^{\kappa_{n}} \mathcal{L}_{n-1}
\end{array}\right),
\end{gathered}
$$

and the integrals with index $a=2$ are obtained by the same rule as in (4.2), (4.3) and (4.4), where the notations $H_{n}^{\kappa_{n}}=H_{n}+\kappa_{n}^{2}$ and $H_{n-1}^{\kappa_{n}}=H_{n-1}+\kappa_{n}^{2}$ are used. To obtain the limit we have taken into account the relations (3.7), (3.12) and (2.16). The Hamiltonian $\mathcal{H}_{n}$ and its integrals of motion generate the nonlinear superalgebra of the form similar to (4.5) and (4.6), but with corresponding changes of the operators on the right hand sides, and with the $\mathcal{C}$ changed for $\kappa_{n}$.

Note that the lower matrix element in the integral $\mathcal{P}_{n, 1}$ (and similarly, in $\mathcal{P}_{n, 2}$ ) is factorized into the subsystem's Hamiltonian $H_{n-1}^{\kappa_{n}}$ and the corresponding Lax-Novikov integral. The multiplicative factor $H_{n-1}^{\kappa_{n}}$ could be omitted there without changing the property of commutativity of the diagonal matrix operators with the Hamiltonian $\mathcal{H}_{n}$. However, this would change the property that the upper and lower matrix elements in the integrals $\mathcal{P}_{n, a}$ are the differential operators of the same order $2 n+1$, and, as a consequence, would complicate the form of the superalgebra.

In spite of a similar form of the superalgebra (with $\mathcal{C}$ changed for $\kappa_{n}$ ), the superextended system we have here is essentially different from the previous one. Indeed, the system $\mathcal{H}_{n}$, unlike the $\breve{\mathcal{H}}_{n}$, possesses now the non-degenerate ground state of zero energy, which corresponds to the lowest bound state of the upper subsystem $H_{n}^{\kappa_{n}}$. This state is annihilated by all the four supercharges and the two bosonic integrals, and the exotic nonlinear supersymmetry we have here corresponds to the unbroken phase. Therefore, the limit we considered provokes the transmutation of the partially broken exotic supersymmetry into the unbroken one.

Also, there exists a limit, associated with the soliton scattering, which transmutes the exotic nonlinear supersymmetry from the unbroken phase into the partially broken exotic supersymmetry. To see this, we apply to the system (4.2), (4.8) the limit $\tau_{n} \rightarrow \infty$, which corresponds to sending the soliton with index $n$ in the subsystem $H_{n}^{\kappa_{n}}$ to infinity. We find then with the help of 3.15 and $(3.16)$ that

$$
\begin{gathered}
\mathcal{H}_{n} \underset{\tau_{n} \rightarrow \infty}{\longrightarrow} \breve{\mathcal{H}}_{n-1}^{\diamond}, \quad \mathcal{S}_{n, a} \underset{\tau_{n} \rightarrow \infty}{\longrightarrow} \breve{\mathcal{S}}_{n-1, a}^{\diamond}, \\
\mathcal{P}_{n, a} \underset{\tau_{n} \rightarrow \infty}{\longrightarrow} \breve{\mathcal{H}}_{n-1}^{\diamond} \breve{\mathcal{P}}_{n-1, a}^{\diamond}, \quad \mathcal{Q}_{n, a} \underset{\tau_{n} \rightarrow \infty}{\longrightarrow}-\breve{\mathcal{H}}_{n-1}^{\diamond} \breve{\mathcal{Q}}_{n-1, a}^{\diamond}+2 \kappa_{n} \breve{\mathbb{P}}_{n-1}^{\diamond} \breve{\mathcal{S}}_{n-1, a}^{\diamond} .
\end{gathered}
$$


Here we have used the notation $F^{\diamond}=\sigma_{2} F \sigma_{2}$, which corresponds to a unitary transformation between the matrix operators

$$
F=\left(\begin{array}{cc}
\alpha & \beta \\
\gamma & \delta
\end{array}\right) \quad \text { and } \quad F^{\diamond}=\left(\begin{array}{cc}
\delta & -\gamma \\
-\beta & \alpha
\end{array}\right),
$$

and imply that the operators indexed by $n-1$ are given by the same expressions as the operators associated with $\breve{\mathcal{H}}_{n}$, but with the parameter $\mathcal{C}$ changed in the structure of the latter operators for $\mathcal{C}=\kappa_{n}$. As a consequence, we also obtain a four-term chain of the limits

$$
\mathcal{H}_{n} \underset{\tau_{n} \rightarrow \infty}{\longrightarrow} \breve{\mathcal{H}}_{n-1}^{\diamond} \underset{\kappa_{n} \rightarrow \kappa_{n-1}}{\longrightarrow} \mathcal{H}_{n-1}^{\diamond} \underset{\tau_{n-1} \rightarrow \infty}{\longrightarrow} \breve{\mathcal{H}}_{n-2} \text {. }
$$

Note that the multiplicative factor $\breve{\mathcal{H}}_{n-1}^{\diamond}$ in the limit of the operators $\mathcal{P}_{n, a}$ and $\mathcal{Q}_{n, a}$ in 4.10 corresponds to a reduction of the order of the integrals that is related with the loss of the one eigenvalue of zero energy in comparison with the spectrum of the system $\mathcal{H}_{n}$.

\section{$5 \quad$ Transparent Dirac systems}

We have discussed the Darboux-Crum transformations, the exotic supersymmetric structure based on them, and transmutations of supersymmetry in the reflectionless systems described by the $2 \times 2$ matrix second order Scrödinger Hamiltonian operators. One can take one of the two first order Hermitian supercharges appearing in these second order systems, and consider it as a first order matrix Hamiltonian for the $(1+1)$-dimensional Dirac system. We can identify then the Darboux-Crum generators, which intertwine such reflectionless first order matrix Hamiltonians. This opens a possibility to investigate exotic supersymmetry and its transmutations in the transparent Dirac systems.

Let us take the first order supercharge $\breve{\mathcal{S}}_{n, 1}$ from $(4.2)$, and identify it as the Dirac Hamiltonian, $\breve{H}_{n}^{D} \equiv \breve{\mathcal{S}}_{n, 1}$. This system corresponds to the $(1+1)$-dimensional Dirac particle in a scalar potential $\Delta_{n}(x)=\Omega_{n}-\tilde{\Omega}_{n}+\mathcal{C}$ with asymptotic behaviour $\Delta_{n}(x) \rightarrow \mathcal{C}$ for $x \rightarrow \pm \infty$. Due to the relation of commutativity $\left[\breve{\mathcal{S}}_{n, 1}, \breve{\mathcal{P}}_{n, 1}\right]=0$, the potentials of this form correspond to the solutions of the multi-kink-antikink type for the stationary mKdV hierarchy [35].

The Dirac Hamiltonian $\breve{H}_{n}^{D}$ has $2 n$ bound states, and its spectrum is symmetric,

$$
\sigma\left(\breve{H}_{n}^{D}\right)=(-\infty,-\mathcal{C}] \cup \breve{\mathcal{E}}_{1}^{-} \cup \ldots \cup \breve{\mathcal{E}}_{n}^{-} \cup \breve{\mathcal{E}}_{n}^{+} \cup \ldots \cup \breve{\mathcal{E}}_{1}^{+} \cup[\mathcal{C}, \infty)
$$

where $\breve{\mathcal{E}}_{i}^{ \pm}= \pm \sqrt{\mathcal{C}^{2}-\kappa_{i}^{2}}, i=1, \ldots, n$, and semi-infinite intervals $[\mathcal{C}, \infty)$ and $(-\infty,-\mathcal{C}]$ correspond to the doubly degenerate continuous parts of the spectrum. In the limit $\mathcal{C} \rightarrow \kappa_{n}$, we have $\breve{H}_{n}^{D} \rightarrow H_{n}^{D}=\mathcal{S}_{n, 1}$, where $\mathcal{S}_{n, 1}$ is defined in (4.7). A scalar potential takes here the form $\mathcal{W}_{n}(x)=\Omega_{n}-\Omega_{n-1}$, with $\mathcal{W}_{n}(x) \rightarrow \mp \kappa_{n}$ for $x \rightarrow \pm \infty$. The potentials of this form are, again, the solutions of the kink (or, antikink) type for the stationary mKdV hierarchy due to the relation $\left[\mathcal{S}_{n, 1}, \mathcal{P}_{n, 1}\right]=0$. The spectrum of the Dirac Hamiltonian $H_{n}^{D}$ has $2 n-1$ bound states, including one bound state of zero energy,

$$
\sigma\left(H_{n}^{D}\right)=\left(-\infty,-\kappa_{n}\right] \cup \mathcal{E}_{1}^{-} \cup \ldots \cup \mathcal{E}_{n-1}^{-} \cup 0 \cup \mathcal{E}_{n-1}^{+} \cup \ldots \cup \mathcal{E}_{1}^{+} \cup\left[\kappa_{n}, \infty\right),
$$

where $\mathcal{E}_{i}^{ \pm}= \pm \sqrt{\kappa_{n}^{2}-\kappa_{i}^{2}}, i=1, \ldots, n-1$. The two discrete energy levels $\breve{\mathcal{E}}_{n}^{-}$and $\breve{\mathcal{E}}_{n}^{+}$of the system $\breve{H}_{n}^{D}$ merge in the limit $\mathcal{C} \rightarrow \kappa_{n}$ and transform into a non-degenerate zero energy level of the bound state for the system $H_{n}^{D}$. 


\subsection{First order matrix Darboux intertwiners for Dirac systems}

Let us return to the identity 3.13,

$$
A_{n}\left(x, \tau_{i}\right) X_{n-1}\left(x, \tau_{i}, \mathcal{C}\right)=X_{n}\left(x, \tau_{i}, \mathcal{C}\right) A_{n}\left(x, \tilde{\tau}_{i}\right),
$$

where $X_{n}\left(x, \tau_{i}, \mathcal{C}\right)=\frac{d}{d x}+\Delta_{n}\left(x, \tau_{i}, \mathcal{C}\right)$, and

$$
\Delta_{n}\left(x, \tau_{i}, \mathcal{C}\right)=\Omega_{n}\left(x, \tau_{i}\right)-\Omega_{n}\left(x, \tilde{\tau}_{i}\right)+\mathcal{C}, \quad \tilde{\tau}_{i}=\tau_{i}-\varphi_{i}(\mathcal{C}), \quad \varphi_{i}(\mathcal{C})=\frac{1}{2 \kappa_{i}} \log \frac{\mathcal{C}+\kappa_{i}}{\mathcal{C}-\kappa_{i}}
$$

If in (5.3) we change $\tau_{i} \rightarrow \tau_{i}+\varphi_{i}(\mathcal{C})$, then make a replacement $\mathcal{C} \rightarrow-\mathcal{C}$, and take into account that $\varphi_{i}(-\mathcal{C})=-\varphi_{i}(\mathcal{C})$ and that $X_{n}$ satisfies the relation $X_{n}\left(x, \tau_{i}-\varphi_{i}(\mathcal{C}),-\mathcal{C}\right)=$ $-X_{n}^{\dagger}\left(x, \tau_{i}, \mathcal{C}\right)$, we obtain the identity

$$
A_{n}\left(x, \tilde{\tau}_{i}\right) X_{n-1}^{\dagger}\left(x, \tau_{i}, \mathcal{C}\right)=X_{n}^{\dagger}\left(x, \tau_{i}, \mathcal{C}\right) A_{n}\left(x, \tau_{i}\right)
$$

Using the notations $A_{n} \equiv A_{n}\left(x, \tau_{i}\right), \tilde{A}_{n}(\mathcal{C}) \equiv A_{n}\left(\tilde{\tau}_{i}\right)$ and $X_{n}(\mathcal{C}) \equiv X_{n}\left(x, \tau_{i}, \mathcal{C}\right)$, the equations (5.3)-(5.5) and their Hermitian conjugate give us the relations

$$
\begin{array}{ll}
A_{n} X_{n-1}=X_{n} \tilde{A}_{n}, & A_{n}^{\dagger} X_{n}=X_{n-1} \tilde{A}_{n}^{\dagger}, \\
\tilde{A}_{n} X_{n-1}^{\dagger}=X_{n}^{\dagger} A_{n}, & \tilde{A}_{n}^{\dagger} X_{n}^{\dagger}=X_{n-1}^{\dagger} A_{n}^{\dagger} .
\end{array}
$$

Using these relations, we can define the intertwining operator between the Dirac Hamiltonians $\breve{H}_{n}^{D}$ and $\breve{H}_{n-1}^{D}$, which also is the intertwining operator between the extended (supersymmetric) Schrödinger Hamiltonians $\breve{\mathcal{H}}_{n}$ and $\breve{\mathcal{H}}_{n-1}$,

$$
\breve{\mathcal{A}}_{n}=\left(\begin{array}{cc}
A_{n} & 0 \\
0 & \tilde{A}_{n}
\end{array}\right), \quad \breve{\mathcal{A}}_{n} \breve{H}_{n-1}^{D}=\breve{H}_{n}^{D} \breve{\mathcal{A}}_{n}, \quad \breve{\mathcal{A}}_{n} \breve{\mathcal{H}}_{n-1}=\breve{\mathcal{H}}_{n} \breve{\mathcal{A}}_{n}
$$

In the limit $\mathcal{C} \rightarrow \kappa_{n}$, the relations in (5.6) are transformed into the trivial identity $A_{n} X_{n-1}\left(\kappa_{n}\right)=A_{n} X_{n-1}\left(\kappa_{n}\right)$, and the relation

$$
A_{n}^{\dagger} A_{n}=X_{n-1}\left(\kappa_{n}\right) X_{n-1}^{\dagger}\left(\kappa_{n}\right)=H_{n-1}+\kappa_{n}^{2},
$$

where we have used the limits (3.7). These identities allow us to construct a new operator of intertwining between the Dirac systems $H_{n}^{D}$ and $\breve{H}_{n-1}^{D}$, and between the super-extended Schrödinger Hamiltonians $\mathcal{H}_{n}$ and $\breve{\mathcal{H}}_{n-1}$,

$$
\mathcal{A}_{n}=\left(\begin{array}{cc}
A_{n} & 0 \\
0 & X_{n-1}\left(\kappa_{n}\right)
\end{array}\right), \quad \mathcal{A}_{n} \breve{H}_{n-1}^{D}\left(\kappa_{n}\right)=H_{n}^{D} \mathcal{A}_{n}, \quad \mathcal{A}_{n} \breve{\mathcal{H}}_{n-1}\left(\kappa_{n}\right)=\mathcal{H}_{n} \mathcal{A}_{n},
$$

where we indicated a dependence of the corresponding operators on $\kappa_{n}=\mathcal{C}$.

This construction corresponds here to the Darboux transformations for reflectionless Dirac systems, and, particularly, gives us a possibility to construct analytically the states of $H_{n}^{D}$ and $\breve{H}_{n}^{D}$ in terms of the eigenstates $\breve{\Phi}_{0}$ of the matrix operator $\breve{H}_{0}^{D}=-\sigma_{2} p+\sigma_{1} \mathcal{C}$

$$
\breve{H}_{0}^{D}(\mathcal{C})=\left(\begin{array}{cc}
0 & \frac{d}{d x}+\mathcal{C} \\
-\frac{d}{d x}+\mathcal{C} & 0
\end{array}\right)
$$


which corresponds to the Hamiltonian of the free massive Dirac particle. The eigenstates $\breve{\Phi}_{n}$ of $\breve{H}_{n}^{D}$ can be presented in the form $\breve{\Phi}_{n}=\breve{\mathcal{A}}_{n} \breve{\mathcal{A}}_{n-1} \ldots \breve{\mathcal{A}}_{1} \breve{\Phi}_{0}$, while the eigenstates of $H_{n}^{D}$ are constructed in the form $\Phi_{n}=\mathcal{A}_{n} \breve{\mathcal{A}}_{n-1} \breve{\mathcal{A}}_{n-2} \ldots \breve{\mathcal{A}}_{1} \breve{\Phi}_{0}$ in terms of the eigenstates $\breve{\Phi}_{0}$ of the Dirac Hamiltonian $\breve{H}_{0}^{D}\left(\kappa_{n}\right)=-\sigma_{2} p+\sigma_{1} \kappa_{n}$. The explicit form of the scattering states and $2 n$ bound states of the $\breve{H}_{n}^{D}$ are given by

$$
\breve{\Phi}_{n}^{\epsilon}\left(\breve{\mathcal{E}}^{ \pm}\left(k^{2}\right)\right)=\left(\begin{array}{c}
\Psi_{n}^{\epsilon}\left(k^{2}\right) \\
\pm \sqrt{\frac{\mathcal{C}-i \epsilon k}{\mathcal{C}+i \epsilon k}} \tilde{\Psi}_{n}^{\epsilon}\left(k^{2}\right)
\end{array}\right), \quad \breve{\Phi}_{n}\left(\breve{\mathcal{E}}_{i}^{ \pm}\right)=\left(\begin{array}{c}
\Psi_{n}\left(-\kappa_{i}^{2}\right) \\
\pm \tilde{\Psi}_{n}\left(-\kappa_{i}^{2}\right)
\end{array}\right)
$$

where $\breve{H}_{n}^{D} \breve{\Phi}_{n}(\breve{\mathcal{E}})=\breve{\mathcal{E}} \breve{\Phi}_{n}(\breve{\mathcal{E}}), \breve{\mathcal{E}}^{ \pm}\left(k^{2}\right)= \pm \sqrt{\mathcal{C}^{2}+k^{2}}, \breve{\mathcal{E}}_{i}^{ \pm}= \pm \sqrt{\mathcal{C}^{2}-\kappa_{i}^{2}}, i=1, \ldots, n, \Psi_{n}$ are Schrödinger eigenstates defined in (2.8), and the parameter $\epsilon= \pm 1$ corresponds to the two possible directions in which the waves can propagate. The two discrete energy levels $\breve{\mathcal{E}}_{n}^{ \pm}= \pm \sqrt{\mathcal{C}^{2}-\kappa_{n}^{2}}$ merge in the limit $\mathcal{C} \rightarrow \kappa_{n}$, and two corresponding eigenstates of $\breve{H}_{n}^{D}$ reduce to the unique state of zero energy of the Dirac Hamiltonian $H_{n}^{D}$,

$$
\breve{\Phi}_{n}\left(\breve{\mathcal{E}}_{n}^{ \pm}\right)=\left(\begin{array}{c}
\Psi_{n}\left(-\kappa_{n}^{2}\right) \\
\pm \tilde{\Psi}_{n}\left(-\kappa_{n}^{2}\right)
\end{array}\right) \rightarrow \Phi_{n}(0)=\left(\begin{array}{c}
\Psi_{n}\left(-\kappa_{n}^{2}\right) \\
0
\end{array}\right)
$$

\subsection{Exotic supersymmetry of reflectionless Dirac systems}

The matrix operator $\breve{\mathcal{P}}_{n, 1}$ and the Dirac Hamiltonian $\breve{\mathcal{H}}_{n}^{D}$ correspond to the Lax pair for the $n$-th member of the stationary mKdV hierarchy, and the scalar Dirac potential $\Delta_{n}(x)$ is identified as the corresponding soliton (multi-kink-antikink type) solution. Since $\left[\breve{\mathcal{P}}_{n, 1}, \breve{\mathcal{H}}_{n}^{D}\right]=$ 0 , the $\breve{\mathcal{P}}_{n, 1}$ is a nontrivial integral for the Dirac system $\breve{H}_{n}^{D}$. It is the Darboux-dressed momentum operator of the free Dirac massive particle (5.11). The interesting point is that for the reflectionless Dirac system $\breve{H}_{n}^{D}$ one can identify an additional integral of motion $\breve{\Gamma}$, which satisfies the identity $\breve{\Gamma}^{2}=1$, and anticommutes with $\breve{\mathcal{P}}_{n, 1}$. As a consequence, the reflectionless Dirac system $\breve{H}_{n}^{D}$ can be characterized by the proper exotic nonlinear supersymmetry. Indeed, consider the operator $\breve{\Gamma}=\mathcal{R} \sigma_{3}$, where $\mathcal{R}$ is the operator of reflection in $x, \tau_{i}$ and $\mathcal{C}$, which satisfies the relations $\mathcal{R} z=-z \mathcal{R}, \mathcal{R}^{2}=1$, where $z=x, \tau_{i}$, or $\mathcal{C}$. Due to the relations $\left[\breve{\Gamma}, \breve{\mathcal{H}}_{n}^{D}\right]=0$ and $\left\{\breve{\Gamma}, \breve{\mathcal{P}}_{n, 1}\right\}=0$, the $\breve{\mathcal{H}}_{n}^{D}$ and $\breve{\mathcal{P}}_{n, 1}$ are identified as bosonic and fermionic operators, respectively. They generate a nonlinear $\mathcal{N}=1$ superalgebra

$$
\left[\breve{\mathcal{P}}_{n, 1}, \breve{\mathcal{H}}_{n}^{D}\right]=0, \quad\left\{\breve{\mathcal{P}}_{n, 1}, \breve{\mathcal{P}}_{n, 1}\right\}=2 \mathbb{P}_{2(2 n+1)}\left(\breve{\mathcal{H}}_{n}^{D}\right)
$$

where

$$
\mathbb{P}_{2(2 n+1)}\left(\breve{\mathcal{H}}_{n}^{D}\right) \equiv\left(\left(\breve{\mathcal{H}}_{n}^{D}\right)^{2}-\mathcal{C}^{2}\right) \prod_{j=1}^{n}\left(\left(\breve{\mathcal{H}}_{n}^{D}\right)^{2}-\left(\mathcal{C}^{2}-\kappa_{j}^{2}\right)\right)^{2}
$$

The $2(n+1)$ zeros of the polynomial in $\mathcal{H}_{n}^{D}$ operator 5.15 correspond to the energies of the singlet states of the reflectionless Dirac system, where $\ddot{\mathcal{E}}_{i}^{ \pm}= \pm \sqrt{\mathcal{C}^{2}-\kappa_{i}}, i=1, \ldots, n$, are the energies of the bound states, while $\pm \mathcal{C}$ correspond to the two singlet states at the edges of the continuous parts of the spectrum ${ }^{6}$. In accordance with $(2.8)$, the left and right moving waves in 5.12 of the scattering sector, which correspond to doubly degenerate energy levels $\breve{\mathcal{E}}^{ \pm}\left(k^{2}\right)$

\footnotetext{
${ }^{6}$ Besides a bound state, each double root $\breve{\mathcal{E}}_{i}^{ \pm}, i=1, \ldots, n$, of the polynomial on left hand side of 5.15 corresponds also to a non-physical eigenstate of $\breve{\mathcal{H}}_{n}^{D}$.
} 
of $\breve{H}_{n}^{D}$ are distinguished by the supercharge $\breve{\mathcal{P}}_{n, 1}$ : they are its eigenstates of the opposite sign egenvalues. Supplementing the integral $\breve{\mathcal{P}}_{n, 1}$ with a (nonlocal) integral $\breve{\mathcal{P}}_{n, 2}=i \breve{\Gamma}_{\mathcal{P}_{n, 1}}$, the $\mathcal{N}=1$ exotic nonlinear supersymmetry of the reflectionless Dirac system $\breve{H}_{n}^{D}$ can be extended to $\mathcal{N}=2:\left\{\breve{\mathcal{P}}_{n, a}, \breve{\mathcal{P}}_{n, b}\right\}=2 \delta_{a b} \mathbb{P}_{2(2 n+1)}\left(\breve{\mathcal{H}}_{n}^{D}\right)$

Applying the limit $\mathcal{C} \rightarrow \kappa_{n}$, we identify the proper exotic supersymmetric structure of $H_{n}^{D}$. In this case, the zero energy eigenstate 5.13 of $H_{n}^{D}$ is also the zero mode of the supercharge $\mathcal{P}_{n, 1}$. In both Dirac reflectionless systems $\breve{H}_{n}^{D}$ and $H_{n}^{D}$, the supercharges detect all the non-degenerate eigenvalues of the Hamiltonians by annihilating the corresponding eigenstates, which are the bound states and the states at the edges of the continuous parts of the spectra. Since the zero energy eigenvalue belongs to the spectrum of $H_{n}^{D}$ but is not present in the spectrum of $\breve{H}_{n}^{D}$, the proper exotic supersymmetry of the Dirac system $\breve{H}_{n}^{D}$ is of the broken nature, while that of $H_{n}^{D}$ corresponds to the unbroken phase. In correspondence with the second relation from (4.9), the limit $\tau_{n} \rightarrow \infty$ applied to the Dirac system $H_{n}^{D}$ with the unbroken proper exotic supersymmetry will produce the system $\breve{H}_{n-1}^{\diamond D}=\breve{\mathcal{S}}_{n-1,1}^{\diamond}$, see Eq. 4.9), characterized by the broken exotic supersymmetry.

\section{Discussion and outlook}

We have considered the two related families of the $(1+1)$ D Dirac reflectionless systems. Each such system corresponds to a fermion in a background of a multi-soliton solution (of the kink, or kink-antikink type) of the mKdV equation. In one of these two families, the $n$-soliton potential $V^{D}(x)=\Delta_{n}(x)$ or $-\Delta_{n}(x)$, where $\Delta_{n}(x)=\Delta_{n}\left(x ; \kappa_{1}, \tau_{1}, \ldots, \kappa_{n}, \tau_{n}, \mathcal{C}\right), \mathcal{C}^{2}>\kappa_{n}^{2}$, is $(2 n+1)$-parametric, while in the second family the potential $V^{D}(x)$ is $2 n$ parametric and corresponds to the function $\mathcal{W}_{n}(x)$ or $-\mathcal{W}_{n}(x)$, where $\mathcal{W}_{n}(x)=\mathcal{W}_{n}\left(x ; \kappa_{1}, \tau_{1}, \ldots, \kappa_{n}, \tau_{n}\right)$. From the viewpoint of the associated extended Schrödinger systems, whose matrix $2 \times 2$ Hamiltonians are given by a square of the corresponding Dirac Hamiltonian $H^{D}=i \sigma_{2} \frac{d}{d x}+$ $\sigma_{1} V^{D}(x)$, the Dirac potential $V^{D}(x)$ is a superpotential. The peculiarity of the considered reflectionless families is that in the case of the supersymmetric Schrödinger systems, in addition to the two first order supercharges $H^{D}$ and $i \sigma_{3} H^{D}$, they are characterized by the two supercharges to be matrix differentials operators of the order $2 n$. Furthermore, they possess two nontrivial bosonic integrals to be differential operators of the order $2 n+1$, which are constructed from the Lax-Novikov integrals of the Schrödinger subsystems. One of these two bosonic integrals is a central charge of the exotic nonlinear superalgebra. The same higher order central charge can be identified as the supercharge (a fermionic generator) of the proper exotic nonlinear supersymmetry of the reflectionless Dirac system. In the case of $V^{D}(x)= \pm \Delta_{n}(x)$, the exotic nonlinear supersymmetries of the Schrödinger and Dirac systems are spontaneously broken, and the quantity $\left(\mathcal{C}^{2}-\kappa_{n}^{2}\right)>0$ measures the scale of the breaking. The choices $V^{D}(x)= \pm \mathcal{W}_{n}(x)$ correspond, on the other hand, to the unbroken exotic supersymmetries. The interesting point is that there exists a limit procedure, admitting the interpretation in the context of a soliton scattering, which relates the two indicated families of the exotic supersymmetric reflectionless systems. One can define a kind of a topological charge by a relation

$$
q=\frac{1}{2\left|V_{0}^{D}\right|} \int_{-\infty}^{\infty} d x \frac{d V^{D}(x)}{d x},
$$


where $V_{0}^{D}=\lim _{x \rightarrow+\infty} V^{D}(x)$. The case of the broken supersymmetry with the kink-antikink type potential $V^{D}(x)= \pm \Delta_{n}(x)$ is characterized then by $q=0$, while the cases of the kink, $V^{D}(x)=-\mathcal{W}_{n}(x)$, and anti-kink, $V^{D}(x)=\mathcal{W}_{n}(x)$, type potentials of the unbroken exotic supersymmetries correspond to $q=+1$ and $q=-1$, respectively. The quantity $2\left|V_{0}^{D}\right|$ gives the gap that separates the upper and lower continuous bands in the spectrum of the Dirac systems, and can be treated as a doubled mass parameter of a fermion in an external scalar potential. The mentioned supercharge of the Dirac system annihilates all its non-degenerate energy states, and being the Dabroux-dressed momentum operator of the free Dirac particle (zero-soliton case), distinguishes the left- and right- moving eigenstates corresponding to the doubly degenerate energy values in the continuum bands of the spectrum.

The described transparent potentials $V^{D}(x)$ appear in many physical applications in the form of stationary solutions for inhomogeneous fermion condensates. Such self-consistent condensates are described by the equations

$$
\left(i \not \partial-V^{D}\right) \psi_{\alpha}=0, \quad V^{D}=-g^{2} \sum_{\alpha=1}^{N} \sum_{\text {occ }} \bar{\psi}_{\alpha} \psi_{\alpha} .
$$

Here the first equation with a generalized flavour index $\alpha=1, \ldots, N$ represents a system of $(1+1) \mathrm{D}$ Dirac equations, the $\sum_{\alpha=1}^{N}$ corresponds to summation in degenerate states, and $\sum_{\text {occ }}$ corresponds to a sum over the completely filled Dirac sea plus a sum over bound states, which usually are partially occupied. Equations (6.1) appear particularly in the superconductivity, in the Gross-Neveu model, and in the physics of conducting polymers. A famous method of solution of (6.1) was realized by Dashen, Hasslacher and Neveu in [3], where this system of equations was rewritten in terms of the scattering data for Schrödinger potentials $U_{ \pm}=\left(V^{D}\right)^{2} \pm \frac{d}{d x} V^{D}-\left(V_{0}^{D}\right)^{2}$, and as a result it was shown that the reflection coefficient for both potentials $U_{ \pm}$has to be equal to zero. For some applications of this result, see [55]-[59]. Using the ideas of supersymmetry, this picture is equivalent to the search of the first order operators $D$ and $D^{\dagger}$, which intertwine and factorize corresponding Schrödinger reflectionless Hamiltonians, $H_{+}=D D^{\dagger}-E_{0}$ y $H_{-}=D^{\dagger} D-E_{0}$. As we have shown, there are only two situations where such a factorization is possible.

- When $H_{+}$and $H_{-}$are completely isospectral, the $V^{D}$ corresponds to the Dirac potentials characterized by the topological charge $q=0$, which are given by inhomogeneous condensates $\pm \Delta_{n}$ with asymptotic behaviour $\Delta_{n} \rightarrow \mathcal{C}$ for $x \rightarrow \pm \infty$.

- In other possible case, the spectra of $H_{+}$and $H_{-}$are different in one bound state only, and inhomogeneous condensate takes here the form $V^{D}=-\mathcal{W}_{n}$ or $V^{D}=\mathcal{W}_{n}$, where $\mathcal{W}_{n} \rightarrow \mp \kappa_{n}$ for $x \rightarrow \pm \infty$, and the topological charge $q$ takes the values +1 or -1 .

On the other hand, the occupation fraction for each non-degenerate state defines the energy of the bound states. Using the method of resolvent, J. Feinberg showed in [4] that for all static condensates the following equality is valid:

$$
\nu_{i}=\frac{2}{\pi} \cot ^{-1}\left(\frac{\kappa_{i}}{\sqrt{\left(V_{0}^{D}\right)^{2}-\kappa_{i}^{2}}}\right), \quad i=1, \cdots n,
$$

where $\nu_{i}$ can take the values $\nu_{i}=0, \frac{1}{N}, \cdots \frac{N-1}{N}, 1$. This result was reproduced in [58] for complex kinks in the context of the Bogoliubov-de Gennes and chiral Gross-Neveu systems. 
The case $N=1, \nu=0,1$ corresponds here to the superconductivity. With these restrictions, the topologically trivial homogeneous condensate is possible, $V^{D}= \pm \Delta_{0}= \pm V_{0}^{D}$, $\nu_{1}=1$ (free Dirac massive particle), as well as the topologically nontrivial inhomogeneous condensate, $V^{D}= \pm \mathcal{W}_{1}, \nu_{1}=0, \kappa_{1}=V_{0}^{D}$.

The case $N=2, \nu=0,1 / 2,1$, corresponds to polymer conductors in the context of the Takayama-Lin-Liu-Maki model [8]; in addition to $V^{D}= \pm \Delta_{0}, \pm \mathcal{W}_{1}$, also the case $\nu_{1}=1 / 2$, $V^{D}= \pm \Delta_{1}, \kappa_{1}=\frac{1}{\sqrt{2}}\left|V_{0}^{D}\right|$ is possible. This last solution is known as a polaron. Other topological solution, which is kink+polaron (or antikink+polaron) corresponds to $V^{D}=$ $\mp \mathcal{W}_{2}\left(\kappa_{1}=\frac{1}{\sqrt{2}}\left|V_{0}^{D}\right|\right.$ and $\left.\kappa_{2}=\left|V_{0}^{D}\right|\right)$.

In the 't Hooft limit $N \rightarrow \infty$, the $\kappa_{i}$ can take any value in the interval $0 \leq \kappa_{i} \leq$ $\left|V_{0}^{D}\right|$, that makes possible to have any stationary soliton solution. So, we see that for the Gross-Neveu model, the Darboux transformations provide a general method to generate real inhomogeneous condensates for 6.1).

Equations (5.8) and (5.10) allow us to obtain a supersymmetric system described by the extended first order matrix Hamiltonian composed from the two Dirac Hamiltonians. In such a way we can get two different families of the extended systems. The first one realizes the unbroken exotic supersymmetry and is given by the Hamiltonian of the form

$$
\mathcal{H}^{D}=\left(\begin{array}{cc}
H_{n}^{D} & \mathbf{0} \\
\mathbf{0} & \breve{H}_{n-1}^{D}
\end{array}\right) .
$$

The matrix integrals for (6.3) given by the first order differential operators are

$$
\mathcal{S}_{1}^{D}=\left(\begin{array}{cc}
\mathbf{0} & \mathcal{A}_{n} \\
\mathcal{A}_{n}^{\dagger} & \mathbf{0}
\end{array}\right), \quad \mathcal{S}_{2}^{D}=i \Sigma_{3} \mathcal{S}_{1}^{D},
$$

where $\Sigma_{3}$ is a $4 \times 4$ diagonal matrix of the form $\Sigma_{3}=\operatorname{diag}\left(1_{2},-1_{2}\right)$ with $1_{2}$ to be the unit $2 \times 2$ matrix. Another family is given by the Hamiltonian of the form

$$
\breve{\mathcal{H}}^{D}=\left(\begin{array}{cc}
\breve{H}_{n}^{D} & \\
\mathbf{0} & \breve{H}_{n-1}^{D}
\end{array}\right)
$$

and its analogous integrals are

$$
\breve{\mathcal{S}}_{1}^{D}=\left(\begin{array}{cc}
\mathbf{0} & \breve{\mathcal{A}}_{n} \\
\breve{\mathcal{A}}_{n}^{\dagger} & \mathbf{0}
\end{array}\right), \quad \breve{\mathcal{S}}_{2}^{D}=i \Sigma_{3} \breve{\mathcal{S}}_{1}^{D} .
$$

The grading operator $\Gamma=\Sigma_{3}$ identifies the extended Dirac Hamiltonians to be bosonic generators, while (6.4) and (6.6) are identified as the fermionic generators. Then we find that the indicated operators satisfy the nonlinear supersymmetry relations to be of the order 2 in corresponding Hamiltonians, $\left\{\mathcal{S}_{a}^{D}, \mathcal{S}_{b}^{D}\right\}=2 \delta_{a b}\left(\mathcal{H}^{D}\right)^{2}$, and $\left\{\breve{\mathcal{S}}_{a}^{D}, \breve{\mathcal{S}}_{b}^{D}\right\}=2 \delta_{a b}\left(\left(\breve{\mathcal{H}}^{D}\right)^{2}-\mathcal{C}^{2}+\kappa_{n}^{2}\right)$. Besides, in each of the two cases, there exist bosonic integrals to be the matrix differential operators of the order $2 n+1$, and fermionic integrals of the order $2 n$.

Also, it is possible to construct supersymmetric Dirac type systems with nonlinear superalgebraic relations of the form $\{S, S\}=2 f\left(\left(\mathcal{H}^{D}\right)^{2}\right)$, where $f$ is a polynomial, by taking in extended Hamiltonian $\mathcal{H}^{D}$ a pair of reflectionless Dirac Hamiltonians with distinct scattering 
data. The picture has to be similar to that obtained in [34] for the reflectionless Schrödinger systems.

We are going to present the detailed investigation of such supersymmetric pictures with extended Dirac Hamiltonians elsewhere.

Note also that the last relations in $(5.8)$ and $(5.10)$ can be used to construct further supersymmetric extensions of the reflectionless Schrödinger systems, in particular, given by $4 \times 4$ matrix Hamiltonians.

Acknowledgements. The work has been partially supported by FONDECYT Grant No. 1130017. A. A. also acknowledges the CONICYT scholarship 21120826.

\section{References}

[1] I. Kay and H. E. Moses, "Reflectionless transmission through dielectrics and scattering potentials," J. Appl. Phys. 27, 1503 (1956).

[2] D. J. Gross and A. Neveu, "Dynamical symmetry breaking in asymptotically free field theories," Phys. Rev. D 10, 3235 (1974).

[3] R. F. Dashen, B. Hasslacher and A. Neveu, "Semiclassical bound states in an asymptotically free theory," Phys. Rev. D 12, 2443 (1975).

[4] J. Feinberg, "All about the static fermion bags in the Gross-Neveu model," Annals Phys. 309, 166 (2004) hep-th/0305240;

J. Feinberg, "On kinks in the Gross-Neveu model," Phys. Rev. D 51, 4503 (1995) hepth/9408120].

[5] N. N. Bogoliubov, "A new method in the theory of superconductivity. I," Sov. Phys. JETP 7, 41 (1958) [Zh. Eksp. Teor. Fiz. 34, 58 (1958)] [Front. Phys. 6, 399 (1961)].

[6] P. G. de Gennes, Superconductivity of Metals and Alloys (Addison-Wesley, Redwood City, CA, 1989).

[7] I. Kosztin, S. Kos, M. Stone, and A. J. Leggett, "Free energy of an inhomogeneous superconductor: A wave-function approach," Phys. Rev. B 58, 9365 (1998);

S. Kos and M. Stone, "Gradient expansion for the free energy of a clean superconductor," Phys. Rev. B 59, 9545 (1999).

[8] H. Takayama, Y. R. Lin-Liu and K. Maki, "Continuum model for solitions in polyacetylene," Phys. Rev. B 21, 2388 (1980).

[9] S. A. Brazovskii and N. N Kirova, "Excitons, polarons, and bipolarons in conducting polymers," JETP Lett. 33, 4 (1981).

[10] W. P. Su, J. R. Schrieffer and A. J. Heeger, "Solitons in polyacetylene," Phys. Rev. Lett. 42, 1698 (1979);

A. J. Heeger, S. Kivelson, J. R. Schrieffer and W. -P. Su, "Solitons in conducting polymers," Rev. Mod. Phys. 60, 781 (1988). 
[11] D. K. Cambell and A. R. Bishop, "Solitons in polyacetylene and relativistic-field-theory models," Phys. Rev. B 24, 4859 (1981).

[12] K. Kim and D.-H. Lee, "Theory of one-dimensional solitons, polarons, and multipolarons: An alternative formulation," Phys. Rev. B 61, 10768 (2000)

[13] A. J. Heeger, S. Kivelson, J. R. Schrieffer and W.-P Su, "Solitons in conducting polymers," Rev. Mod. Phys. 60, 781 (1988).

[14] R. Jackiw and C. Rebbi, "Solitons with fermion number 1/2," Phys. Rev. D 13, 3398 (1976);

R. Jackiw and J. R. Schrieffer, "Solitons with fermion number 1/2 in condensed matter and relativistic field theories," Nucl. Phys. B 190, 253 (1981).

[15] J. Goldstone and F. Wilczek, "Fractional quantum numbers on solitons," Phys. Rev. Lett. 47, 986 (1981).

[16] A. J. Niemi and G. W. Semenoff, "Fermion number fractionization in quantum field theory," Phys. Rept. 135, 99 (1986);

G. W. Semenoff and P. Sodano, "Stretching the electron as far as it will go," Electron. J. Theor. Phys. 10, 157 (2006) cond-mat/0605147].

[17] R. Jackiw, "Fractional charge and zero modes for planar systems in a magnetic field," Phys. Rev. D 29, 2375 (1984) [Erratum-ibid. D 33, 2500 (1986)].

[18] M. Thies, "From relativistic quantum fields to condensed matter and back again: Updating the Gross-Neveu phase diagram," J. Phys. A 39, 12707 (2006) hep-th/0601049.

[19] J. Bar-Sagi and C. G. Kuper, "Self-consistent pair potential in an inhomogeneous superconductor," Phys. Rev. Lett. 28, 1556 (1972);

J. Bar-Sagi and C. G. Kuper, "Self-consistent pair potential in superconductors. I. The superconductor-insulator boundary near $T_{c}, "$ J. Low Temp. Phys. 16, 73 (1974).

[20] T. Yefsah, A. T. Sommer, M. J.H. Ku, L. W. Cheuk, W. Ji, W. S. Bakr, and M. W. Zwierlein, "Heavy solitons in a fermionic superfluid," Nature 499, 426 (2013).

[21] T. L. Ho, J. R. Fulco, J. R. Schrieffer and F. Wilczek, "Solitons in superfluid He-3-A: Bound states on domain walls," Phys. Rev. Lett. 52, 1524 (1984).

[22] A. F. Andreev, "Thermal conductivity of the intermediate state of superconductors," Zh. Eksp. Teor. Fiz. 46, 1823 (1964) [JETP 19, 1228 (1964)];

J. Bardeen, R. Kümmel, A. E. Jacobs, and L. Tewordt, "Structure of vortex lines in pure superconductors," Phys. Rev. 187, 556 (1969).

[23] E. Witten and D. I. Olive, "Supersymmetry algebras that include topological charges," Phys. Lett. B 78, 97 (1978).

[24] E. Witten, "Dynamical breaking of supersymmetry," Nucl. Phys. B 188, 513 (1981); E. Witten, "Constraints on supersymmetry breaking," Nucl. Phys. B 202, 253 (1982). 
[25] F. Cooper, A. Khare and U. Sukhatme, "Supersymmetry and quantum mechanics," Phys. Rept. 251, 267 (1995) hep-th/9405029.

[26] J. D. Edelstein, M. L. Trobo, F. A. Brito and D. Bazeia, "Kinks inside supersymmetric domain ribbons," Phys. Rev. D 57, 7561 (1998) [hep-th/9707016].

[27] G. R. Dvali and M. A. Shifman, "Domain walls in strongly coupled theories," Phys. Lett. B 396, 64 (1997) [Erratum-ibid. B 407, 452 (1997)] hep-th/9612128.

[28] M. A. Shifman, A. I. Vainshtein and M. B. Voloshin, "Anomaly and quantum corrections to solitons in two-dimensional theories with minimal supersymmetry," Phys. Rev. D 59, 045016 (1999) [hep-th/9810068.

[29] V. B. Matveev and M. A. Salle, Darboux Transformations and Solitons (Springer, Berlin, 1991).

[30] H. D. Wahlquist and F.B. Estabrook, "Bäcklund transformations for solution of the Korteweg-de Vries equation," Phys. Rev. Lett. 31, 1386 (1973).

[31] P. Drazin and R. Johnson, Solitons: An Introduction (Cambridge University Press, Cambridge, England, 1996).

[32] S. P. Novikov, "The periodic problem for the Korteweg-de Vries equation," Funct. Anal. Appl. 8, 236 (1975).

[33] S. P. Novikov, S.V. Manakov, L. P. Pitaevskii, and V. E. Zakharov, Theory of Solitons (Plenum, New York, 1984).

[34] A. Arancibia, J. M. Guilarte and M. S. Plyushchay, "Effect of scalings and translations on the supersymmetric quantum mechanical structure of soliton systems," Phys. Rev. D 87, 045009 (2013) arXiv:1210.3666 [math-ph]].

[35] A. Arancibia, J. M. Guilarte and M. S. Plyushchay, "Fermion in a multi-kink-antikink soliton background, and exotic supersymmetry," Phys. Rev. D 88, 085034 (2013) arXiv:1309.1816 [hep-th]].

[36] M. S. Plyushchay, A. Arancibia and L. -M. Nieto, "Exotic supersymmetry of the kink-antikink crystal, and the infinite period limit," Phys. Rev. D 83, 065025 (2011) arXiv:1012.4529 [hep-th]].

[37] V. E. Zakharov and A. B. Shabat, "Exact theory of two-dimensional self-focusing and one-dimensional self-modulation of waves in nonlinear media," Zh. Eksp. Teor. Fiz. 61, 118 (1971) [Sov. Phys. JETP 34, 62 (1972)].

[38] M. J. Ablowitz, D. J. Kaup, A. C. Newell and H. Segur, "Nonlinear-evolution equations of physical significance," Phys. Rev. Lett. 31, 125 (1973);

M. J. Ablowitz, D. J. Kaup, A. C. Newell and H. Segur, "The Inverse scattering transform fourier analysis for nonlinear problems," Stud. Appl. Math. 53, 249 (1974). 
[39] C. S. Gardner, J. Greene, M. Kruskal, and R. Miura, "Method for solving the Kortewegde Vries equation," Phys. Rev. Lett. 19, 1095 (1967).

[40] R. M. Miura, "Korteweg-deVries equation and generalizations. I. A remarkable explicit nonlinear transformation," J . Math. Phys. 9, 1202 (1968).

[41] J. L. Burchnall and T. W. Chaundy, "Commutative ordinary differential operators," Proc. London Math. Soc. Ser. 2, s2-21, 420 (1923);

I. M. Krichever, "Commutative rings of ordinary linear differential operators," Funct. Anal. Appl. 12, 175 (1978).

[42] M. S. Plyushchay and L. -M. Nieto, "Self-isospectrality, mirror symmetry, and exotic nonlinear supersymmetry," Phys. Rev. D 82, 065022 (2010) [arXiv:1007.1962 [hep-th]].

[43] M. S. Plyushchay, "Deformed Heisenberg algebra, fractional spin fields and supersymmetry without fermions," Annals Phys. 245, 339 (1996) hep-th/9601116];

M. S. Plyushchay, "Hidden nonlinear supersymmetries in pure parabosonic systems," Int. J. Mod. Phys. A 15, 3679 (2000) [hep-th/9903130];

V. Jakubsky, L. -M. Nieto and M. S. Plyushchay, "The origin of the hidden supersymmetry," Phys. Lett. B 692, 51 (2010) [arXiv:1004.5489 [hep-th]].

[44] F. Correa, V. Jakubsky, L. -M. Nieto and M. S. Plyushchay, "Self-isospectrality, special supersymmetry, and their effect on the band structure," Phys. Rev. Lett. 101, 030403 (2008) arXiv:0801.1671 [hep-th]];

F. Correa, V. Jakubsky and M. S. Plyushchay, "Finite-gap systems, tri-supersymmetry and self-isospectrality," J. Phys. A 41, 485303 (2008) [arXiv:0806.1614 [hep-th]];

F. Correa, V. Jakubsky and M. S. Plyushchay, "Aharonov-Bohm effect on AdS(2) and nonlinear supersymmetry of reflectionless Poschl-Teller system," Annals Phys. 324, 1078 (2009) |arXiv:0809.2854 [hep-th]].

[45] F. Correa, G. V. Dunne and M. S. Plyushchay, "The Bogoliubov-de Gennes system, the AKNS hierarchy, and nonlinear quantum mechanical supersymmetry," Annals Phys. 324, 2522 (2009) [arXiv:0904.2768 [hep-th]].

[46] F. Gesztesy, W. Schweiger and B. Simon, "Commutation Methods Applied to the mKdVEquation," Trans. Amer. Math. Soc. 324, 465 (1991).

[47] A. P. Veselov and A. B. Shabat, "Dressing chains and the spectral theory of the Schrödinger operator," Funct. Anal. Appl., 27 (2), 81 (1993).

[48] F. Gesztesy and R. Weikard, "Lamé potentials and the stationary $(m) K d V$ hierarchy,", Mathematische Nachrichten 176, 73 (1995).

[49] D. J. Fernandez C., B. Mielnik, O. Rosas-Ortiz and B. F. Samsonov, "Nonlocal SUSY deformations of periodic potentials," J. Phys. A 35, 4279 (2002) quant-ph/0303051; "The Phenomenon of Darboux displacements," Phys. Lett. A 294, 168 (2002) |quantph/0302204. 
[50] A. A. Andrianov and A. V. Sokolov, "Nonlinear supersymmetry in quantum mechanics: Algebraic properties and differential representation," Nucl. Phys. B 660 (2003) 25 hepth/0301062.

[51] D. A. Takahashi, S. Tsuchiya, R. Yoshii and M. Nitta, "Fermionic solutions of chiral Gross-Neveu and Bogoliubov-de Gennes systems in nonlinear Schrödinger hierarchy," Phys. Lett. B 718, 632 (2012) [arXiv:1205.3299 [cond-mat.supr-con]].

[52] A. Klotzek and M. Thies, "Kink dynamics, sinh-Gordon solitons and strings in $A d S_{3}$ from the Gross-Neveu model," J. Phys. A 43, 375401 (2010) [arXiv:1006.0324 [hep-th]].

[53] G. A. El, "Korteweg-de Vries equation: solitons and undular bores," In: Solitary Waves in Fluids, Advaces in Fluid Mechanics, Vol. 47, WIT Press, UK (2007); ed. by R. Grimshaw.

[54] A. A. Andrianov and M. V. Ioffe, "Nonlinear supersymmetric quantum mechanics: concepts and realizations," J. Phys. A 45 (2012) 503001 [arXiv:1207.6799 [hep-th]].

[55] V. Schon and M. Thies, "Emergence of Skyrme crystal in Gross-Neveu and 't Hooft models at finite density," Phys. Rev. D 62, 096002 (2000) [hep-th/0003195].

[56] J. Feinberg and S. Hillel, "Stable fermion bag solitons in the massive Gross-Neveu model: Inverse scattering analysis," Phys. Rev. D 72, 105009 (2005) [hep-th/0509019].

[57] G. Basar and G. V. Dunne, "Self-consistent crystalline condensate in chiral GrossNeveu and Bogoliubov-de Gennes systems," Phys. Rev. Lett. 100, 200404 (2008) arXiv:0803.1501 [hep-th]];

G. Basar and G. V. Dunne, "Gross-Neveu models, nonlinear Dirac equations, surfaces and strings," JHEP 1101, 127 (2011) |arXiv:1011.3835 [hep-th]];

G. Basar, G. V. Dunne and M. Thies, "Inhomogeneous condensates in the thermodynamics of the chiral NJL(2) model," Phys. Rev. D 79, 105012 (2009) arXiv:0903.1868 [hep-th]].

[58] D. A. Takahashi and M. Nitta, "Self-consistent multiple complex-kink solutions in Bogoliubov-de Gennes and chiral Gross-Neveu systems," Phys. Rev. Lett. 110, 131601 (2013) arXiv:1209.6206 [cond-mat.supr-con]].

[59] G. V. Dunne and M. Thies, "Time-dependent Hartree-Fock solution of Gross-Neveu models: Twisted kink constituents of baryons and breathers," Phys. Rev. Lett. 111, 121602 (2013) [arXiv:1306.4007 [hep-th]];

G. V. Dunne and M. Thies, "Transparent Dirac potentials in one dimension: the timedependent case," Phys. Rev. A 88, 062115 (2013) [arXiv:1308.5801 [hep-th]]. 